\title{
THE HOCHSCHILD COHOMOLOGY ALGEBRA FOR A FAMILY OF SELF-INJECTIVE ALGEBRAS OF THE TREE CLASS $D_{n}$
}

\author{
YU. V. VOLKOV
}

\begin{abstract}
The Hochschild cohomology algebra for a series of self-injective algebras of the tree class $D_{n}$ is described in terms of generators and relations. The proof involves the existing description of the minimal bimodule resolvent and of the additive structure for the Hochschild cohomology algebras in question.
\end{abstract}

\section{§1. INTRODUCTION}

Let $R$ be a representation-finite self-injective basic algebra over an algebraically closed field. The stable $A R$-quiver of such an algebra can be described with the help of an associated tree, which coincides with one of the Dynkin diagrams $A_{n}, D_{n}, E_{6}, E_{7}$, or $E_{8}$ (see [1]). It is known [2] that the Hochschild cohomology of the algebra $R$ is periodic. If the associated tree of $R$ has type $A_{n}$, then, by [3], $R$ is stably equivalent to a serial selfinjective algebra, or to the so-called "Möbius algebra". In [4, the Hochschild cohomology algebra $\mathrm{HH}^{*}(R)$ for serial self-injective algebras was calculated. Furthermore, for the Möbius algebra $R$, the subalgebra $\mathrm{HH}^{* r}(R)$ of $\mathrm{HH}^{*}(R)$ was calculated in [5], where $\mathrm{HH}^{* r}(R)$ is the subalgebra generated by homogeneous elements of degree divisible by $r$; here $r$ is some parameter linked with the defining relations for $R$. In [4, 5], the following fact was used substantially: a syzygy of an appropriate order for the $R$-bimodule $R$ can be described as a twisted bimodule.

A more direct approach to the calculation of the Hochschild cohomology for the Möbius algebra $R$ was suggested in [6]. Namely, in that paper the minimal projective resolution was constructed for the algebra $R$ viewed as a $\Lambda$-module, where $\Lambda$ is the enveloping algebra of $R$, and then, in [7, this resolution was used for the description of an additive structure of the algebra $\mathrm{HH}^{*}(R)$, i.e., the dimensions of the groups $\mathrm{HH}^{t}(R)$ were calculated for the Möbius algebra $R$.

If the algebra $R$ is of the tree class $D_{n}$, then, by [8, $R$ is stably equivalent to an algebra of one of five types. Their bound quivers were presented also in [8]. In [9], the approach of [6] was employed to construct the periodic minimal projective resolution for the algebras of one of these types. Then it was used for the calculation of the additive structure of $\mathrm{HH}^{*}(R)$. The present paper completes the description of the Hochschild cohomology algebra for the algebras considered in [9. Using the description of the bimodule resolution and the additive structure of the Hochschild cohomology algebra, we find a finite set of generators of $\mathrm{HH}^{*}(R)$ and the relations they satisfy.

\section{§2. Bimodule Resolution and the Additive structure of the Hochschild COHOMOLOGY ALGEBRA}

In this section, we recall the notation and results from 9 .

2010 Mathematics Subject Classification. Primary 13D03.

Key words and phrases. Hochschild cohomology, finite type representations, self-injective algebras.

Supported by RFBR (grant no. 10-01-00635a). 
Assume that $k$ is an algebraically closed field. We consider the following bound quiver $(Q, I)$. The set of vertices is $Q_{0}=\{i \in \mathbb{N} \mid 1 \leq i \leq r n\}$; in the sequel, the elements of $Q_{0}$ are specified modulo $\mathrm{rn}$. The set of arrows $Q_{1}$ in the quiver $Q$ consists of the elements

$$
\alpha_{s, i}:(s-1) n+i \rightarrow(s-1) n+i+1,
$$

where $s \in\{1, \ldots, r\}, i \in\{1, \ldots, n-3\}$, and

$$
\begin{aligned}
& \gamma_{s, n-1}: s n-2 \rightarrow s n-1, \gamma_{s, n}: s n-2 \rightarrow s n, \\
& \beta_{s, n-1}: s n-1 \rightarrow s n+1, \beta_{s, n}: s n \rightarrow s n+1,
\end{aligned}
$$

where $s \in\{1, \ldots, r\}$. In this notation for arrows, the first index is determined modulo $r$.
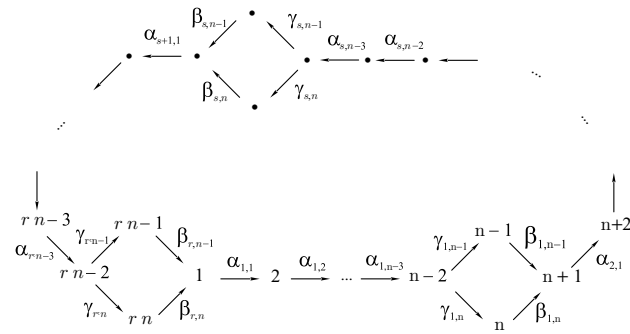

The ideal $I$ is generated by the following elements of the path algebra $k Q$ of $Q$ :

$$
\begin{aligned}
& \gamma_{s, n-1} \alpha_{s, n-3} \ldots \alpha_{s, 1} \beta_{s-1, n} \text { and } \gamma_{s, n} \alpha_{s, n-3} \ldots \alpha_{s, 1} \beta_{s-1, n-1}, \\
& \beta_{s, n-1} \gamma_{s, n-1}-\beta_{s, n} \gamma_{s, n}, \\
& \alpha_{s+1, t} \ldots \alpha_{s+1,1} \beta_{s, n-1} \gamma_{s, n-1} \alpha_{s, n-3} \ldots \alpha_{s, t},
\end{aligned}
$$

where $s=1, \ldots, r, t=1, \ldots, n-3$. We consider the $k$-algebra $R=k Q / I$ and denote by $\Lambda=R \otimes R^{\text {op }}$ the enveloping algebra of $R$. Let $e_{i, j}(1 \leq i \leq r, 1 \leq j \leq n)$ be the idempotent of $R$ corresponding to the vertex $(i-1) n+j$ of the quiver $Q$. In the sequel, the first index in $e_{i, j}$ will be taken modulo $r$. Then $\left\{e_{i_{1}, j_{1}} \otimes e_{i_{2}, j_{2}}\right\}_{i_{1}, j_{1}, i_{2}, j_{2}}$ is a full set of orthogonal primitive idempotents of the algebra $\Lambda$. Let $P_{i, j}=R e_{i, j}$ be the projective $R$-module corresponding to the vertex $(i-1) n+j$ of the quiver $Q$ and $S_{i, j}$ the corresponding simple $R$-module. We denote by $P_{\left[i_{1}, j_{1}\right]\left[i_{2}, j_{2}\right]}=\Lambda e_{i_{1}, j_{1}} \otimes e_{i_{2}, j_{2}}$ the projective $\Lambda$-module corresponding to the idempotent $e_{i_{1}, j_{1}} \otimes e_{i_{2}, j_{2}}$. If $w$ is a path in $Q$ starting at the vertex $\left(i_{1}-1\right) n+j_{1}$ and ending at the vertex $\left(i_{2}-1\right) n+j_{2}$, then right multiplication by $w$ induces a homomorphism $w^{*}: P_{i_{2}, j_{2}} \rightarrow P_{i_{1}, j_{1}}$. For simplicity, we denote this homomorphism also by $w$. If $w_{1}$ is a path starting at the vertex $\left(i_{1}-1\right) n+j_{1}$ and ending at the vertex $\left(i_{2}-1\right) n+j_{2}$, and $w_{2}$ is a path starting at the vertex $\left(i_{3}-1\right) n+j_{3}$ and ending at the vertex $\left(i_{4}-1\right) n+j_{4}$, then $w_{1} \otimes w_{2} \in \operatorname{Hom}_{\Lambda}\left(P_{\left[i_{2}, j_{2}\right]\left[i_{3}, j_{3}\right]}, P_{\left[i_{1}, j_{1}\right]\left[i_{4}, j_{4}\right]}\right)$.

We introduce the following auxiliary notation:

$$
\tau_{i}=\beta_{i, n} \gamma_{i, n}, \quad \mu_{i, j}=\alpha_{i, j} \ldots \alpha_{i, 1}, \quad \nu_{i, j}=\alpha_{i, n-3} \ldots \alpha_{i, j} .
$$

Here, for uniformity reasons, we assume that an empty product of arrows is identified with an appropriate idempotent of the algebra $R$; for example, $\mu_{i, 0}=e_{i, 1}, \alpha_{i, j-1} \ldots \alpha_{i, j}=$ $e_{i, j}, \nu_{i, n-2}=e_{i, n-2}$.

We introduce a $k$-linear map $\sigma: R \rightarrow R$ such that $\sigma(x y)=\sigma(x) \sigma(y)$ and

$$
\begin{aligned}
& \sigma\left(e_{i, j}\right)=e_{i+n-1, \phi^{n}(j)}, \quad \sigma\left(\alpha_{i, j}\right)=\alpha_{i+n-1, j}, \quad \sigma\left(\gamma_{i, n-1}\right)=-\gamma_{i+n-1, \phi^{n}(n-1)}, \\
& \sigma\left(\gamma_{i, n}\right)=-\gamma_{i+n-1, \phi^{n}(n)}, \quad \sigma\left(\beta_{i, n-1}\right)=\beta_{i+n-1, \phi^{n}(n-1)}, \quad \sigma\left(\beta_{i, n}\right)=\beta_{i+n-1, \phi^{n}(n)},
\end{aligned}
$$


where $\phi(j)=j$ for $1 \leq j \leq n-2, \phi(n-1)=n$, and $\phi(n)=n-1$. Clearly, $\sigma$ is an automorphism of the algebra $R$.

In [9], it was shown that $\sigma$ is a finite order automorphism of $R$. This order was calculated in the same paper. In the sequel, we use the notation

$$
\lambda=(2 n-3) \operatorname{ord}(\sigma)
$$

Consider the following modules:

$$
\begin{aligned}
& Q_{2 m}= \bigoplus_{i=1}^{r}\left(\left(\bigoplus_{j=1}^{n-2-m} P_{[i+m, j+m][i, j]}\right) \oplus\left(\bigoplus_{j=n-1-m}^{n-2} P_{[i+m, j+m-(n-2)][i, j]}\right)\right. \\
&\left.\oplus P_{\left[i+m, \phi^{m}(n-1)\right][i, n-1]} \oplus P_{\left[i+m, \phi^{m}(n)\right][i, n]}\right)(m=0, \ldots, n-2), \\
& Q_{2 m+1}= \bigoplus_{i=1}^{r}\left(\left(\bigoplus_{j=1}^{n-3-m} P_{[i+m, j+m+1][i, j]}\right) \oplus P_{[i+m, n-1][i, n-2-m]} \oplus P_{[i+m, n][i, n-2-m]}\right. \\
& \oplus\left.\left(\bigoplus_{j=n-1-m}^{n-2} P_{[i+m+1, j+m-(n-2)][i, j]}\right) \oplus P_{[i+m+1, m+1][i, n-1]} \oplus P_{[i+m+1, m+1][i, n]}\right) \\
&(m=0, \ldots, n-3) .
\end{aligned}
$$

Let $\rho \Lambda$ be the category of finitely generated projective (left) $\Lambda$-modules. We introduce the $k$-linear functor $\sigma: \rho \Lambda \rightarrow \rho \Lambda$. Let $V=\bigoplus_{l \in L} P_{\left[i_{2, l}, j_{2, l}\right]\left[i_{1, l}, j_{1, l}\right]}$. Then $\sigma(V)=\bigoplus_{l \in L} P_{\left[\sigma\left(i_{2, l}, j_{2, l}\right)\right]\left[i_{1, l}, j_{1, l}\right]}$ (here, $\left.\sigma(i, j)=(x, y) \Leftrightarrow \sigma\left(e_{i, j}\right)=e_{x, y}\right)$. Assume that $V_{1}$ and $V_{2}$ are modules in $\rho \Lambda, V_{1}=\bigoplus_{l \in L} P_{\left[i_{2, l}, j_{2 l}\right]\left[i_{1, l}, j_{1, l}\right]}$. Let $d: V_{1} \rightarrow V_{2}$ be a homomorphism of $\Lambda$-modules such that $\left.d\right|_{\left.\left.P_{\left[i_{2}, l\right.}, j_{2, l]}\right] i_{1, l}, j_{1, l}\right]}=\sum_{t \in T} u_{l, t} w_{l, t, 1} \otimes w_{l, t, 2}$, where $u_{l, t} \in k$, $w_{l, t, 1}, w_{l, t, 2} \in R$. Then we define $\left.\sigma(d)\right|_{P_{\left[\sigma\left(i_{2, l}, j_{2, l}\right)\right]\left[i_{1, l}, j_{1, l}\right]}}=\sum_{t \in T} u_{l, t} \sigma\left(w_{l, t, 1}\right) \otimes w_{l, t, 2}$.

Let $\mu: Q_{0} \rightarrow R$ be a homomorphism defined as follows: for $w_{1} \otimes w_{2} \in P_{[i, j][i, j]}$, we put $\mu\left(w_{1} \otimes w_{2}\right)=w_{1} w_{2}$. The homomorphisms $d_{i}: Q_{i+1} \rightarrow Q_{i}$ for $0 \leq i \leq 2 n-4$ (with $\left.Q_{2 n-3}=\sigma\left(Q_{0}\right)\right)$ were described in [9]. The following theorem was proved in the same paper.

Theorem 1. The minimal $\Lambda$-projective resolution of the module $R$ is presented by the following sequence:

$$
0 \leftarrow R \stackrel{\mu}{\longleftarrow} Q_{0} \stackrel{d_{0}}{\longleftarrow} Q_{1} \stackrel{d_{1}}{\longleftarrow} \cdots \stackrel{d_{2 n-5}}{\longleftarrow} Q_{2 n-4} \stackrel{d_{2 n-4}}{\longleftarrow} Q_{2 n-3} \stackrel{d_{2 n-3}}{\longleftarrow} Q_{2 n-2} \stackrel{d_{2 n-2}}{\longleftarrow} \cdots,
$$

where the modules $Q_{i}$ for $i=0, \ldots, 2 n-3$ are as described above, and the homomorphisms $d_{i}$ for $i=0, \ldots, 2 n-4$ are as mentioned before this theorem. Moreover, for $0 \leq t \leq 2 n-4$ we put $Q_{l(2 n-3)+t}=\sigma^{l}\left(Q_{t}\right), d_{l(2 n-3)+t}=\sigma^{l}\left(d_{t}\right)$.

Furthermore, in the same paper, the following two theorems were proved, in which the additive structure of the Hochschild cohomology algebra of $R$ was described for $r>1$ and $r=1$, respectively.

Theorem 2. Let $\mathrm{HH}^{s}(R)$ be the sth group of Hochschild cohomology of the algebra $R$ with coefficients in $R$. Assume that $r>1$. Then 
$\operatorname{dim}_{k} \mathrm{HH}^{s}(R)=1$ if one of the following conditions is fulfilled:

$\left\{\begin{array}{l}s \in\{2 m+l(2 n-3), 2 m+1+l(2 n-3)\}, 0 \leq m \leq n-3, \\ r|m+l(n-1), 2| m+l n \text { and either char } k=2 \text { or } 2 \mid l ;\end{array}\right.$

$\left\{\begin{array}{l}s \in\{2 m+1+l(2 n-3), 2 m+2+l(2 n-3)\}, 0 \leq m \leq n-3, \\ r \mid m+l(n-1), 2 \nmid m+l n \text { and either char } k=2 \text { or } 2 \nmid l ;\end{array}\right.$

$s \in\{(l+1)(2 n-3)-1,(l+1)(2 n-3)\}, r \mid(l+1)(n-1)-1$,

$2 \nmid \ln , \operatorname{char} k=2$;

$s \in\{(l+1)(2 n-3)-1,(l+1)(2 n-3)\}, r \mid(l+1)(n-1)-1$,

$2 \nmid(l+1) n$;

$\operatorname{dim}_{k} \mathrm{HH}^{s}(R)=2$ if

$$
\begin{aligned}
& s \in\{(l+1)(2 n-3)-1,(l+1)(2 n-3)\}, r \mid(l+1)(n-1)-1, \\
& 2 \mid n, \text { and either char } k=2 \text { or } 2 \mid l
\end{aligned}
$$

and $\operatorname{dim}_{k} \mathrm{HH}^{s}(R)=0$ if none of the above conditions holds true.

Theorem 3. Assume that $r=1$. Then

$\operatorname{dim}_{k} \mathrm{HH}^{s}(R)=1$ if one of the following conditions is fulfilled:

$$
\begin{aligned}
& s \in\{2(n-2)+l(2 n-3),(l+1)(2 n-3)\}, 2 \nmid l, \quad \text { and } \text { char } k \neq 2 ; \\
& s \in\{2(n-2)+l(2 n-3),(l+1)(2 n-3)\}, 2 \nmid(l+1) n ; \\
& s=2 m+1+l(2 n-3), 0 \leq m \leq n-3,2 \nmid m+l n, \quad \text { and } \\
& \text { either } 2 \nmid l \text { or } \operatorname{char} k=2 ; \\
& s=2 m+1+l(2 n-3), 0 \leq m \leq n-3,2 \mid m+l n, \quad \text { and } \\
& \text { either } 2 \mid l \text { or char } k=2 ; \\
& s=2 m+l(2 n-3), 1 \leq m \leq n-3,2 \mid m+\ln , \quad \text { and } \quad \text { char } k \neq 2 ;
\end{aligned}
$$

$\operatorname{dim}_{k} \mathrm{HH}^{s}(R)=2$ if one of the following conditions is fulfilled:

$$
\begin{aligned}
& s \in\{2(n-2)+l(2 n-3),(l+1)(2 n-3)\}, 2|n, 2| l, \quad \text { and } \quad \operatorname{char} k \neq 2 ; \\
& s \in\{2(n-2)+l(2 n-3),(l+1)(2 n-3)\}, 2 \nmid l n, \quad \text { and } \quad \operatorname{char} k=2 ; \\
& s=2 m+l(2 n-3), 1 \leq m \leq n-3,2 \mid m+l n, \quad \text { and } \quad \text { char } k=2 ;
\end{aligned}
$$

$\operatorname{dim}_{k} \mathrm{HH}^{s}(R)=3$ if

$$
s \in\{2(n-2)+l(2 n-3),(l+1)(2 n-3)\}, 2 \mid n, \quad \text { and } \quad \operatorname{char} k=2 ;
$$

$\operatorname{dim}_{k} \mathrm{HH}^{s}(R)=n+1$ if $s=0$;

and $\operatorname{dim}_{k} \mathrm{HH}^{s}(R)=0$ if none of the above conditions holds true.

\section{$\S 3$. Calculation of translates}

We put $\delta^{s}=\operatorname{Hom}_{\Lambda}\left(d_{s}, R\right): \operatorname{Hom}_{\Lambda}\left(Q_{s}, R\right) \rightarrow \operatorname{Hom}_{\Lambda}\left(Q_{s+1}, R\right)$, where $d_{s}$ is the differential in the resolution described in Theorem 1 (see also [9, 127-130]).

Any $s$-cycle $f \in \operatorname{Ker} \delta^{s}$ can be lifted (uniquely up to homotopy) to a chain map of complexes $\left\{\phi_{t}: Q_{s+t} \rightarrow Q_{t}\right\}_{t \geq 0}$. The homomorphism $\phi_{t}$ is called the $t$ th translate of $f$ and will be denoted by $T_{t}(f)$. For any cocycles $f \in \operatorname{Ker} \delta^{s}$ and $g \in \operatorname{Ker} \delta^{t}$, we have

$$
\operatorname{cl} g \cdot \operatorname{cl} f=\operatorname{cl}\left(\mu T_{0}(g) T_{t}(f)\right) .
$$

Hence, we need formulas for translates of some elements of the Hochschild cohomology algebra to deduce relations in this algebra. This section is devoted to the calculation of translates needed for the description of relations in $\mathrm{HH}^{*}(R)$. 
Remark 1. In the sequel, we usually denote by $f$ the cohomology class $\mathrm{cl} f \in \mathrm{HH}^{s}(R)$ of the $s$-cocycle $f \in \operatorname{Ker} \delta^{s}$.

Let $w$ be a path from the vertex $\left(i_{1}-1\right) n+j_{1}$ to the vertex $\left(i_{2}-1\right) n+j_{2}$. We denote by $w^{*}$ the $\Lambda$-homomorphism belonging to $\operatorname{Hom}_{\Lambda}\left(P_{\left[i_{2}, j_{2}\right]\left[i_{1}, j_{1}\right]}, R\right)$ such that $w^{*}\left(e_{i_{2}, j_{2}} \otimes\right.$ $\left.e_{i_{1}, j_{1}}\right)=w$. For any $1 \leq i \leq r$, we introduce the following auxiliary homomorphisms:

$$
\begin{array}{rlrl}
w_{i, m, j} & =\left(\alpha_{i, j+m-1} \ldots \alpha_{i, j}\right)^{*} & (0 \leq m \leq n-3,1 \leq j \leq n-2-m) ; \\
t_{i, m, j} & =\left(\mu_{i+1, j+m-(n-1)} \tau_{i} \nu_{i, j}\right)^{*} & (1 \leq m \leq n-2, n-1-m \leq j \leq n-2) ; \\
u_{i, m, q} & =\left(\gamma_{i, q} \nu_{i, n-2-m}\right)^{*} & (0 \leq m \leq n-3, q \in\{n-1, n\}) ; \\
v_{i, m, q} & =\left(\mu_{i+1, m} \beta_{i, q}\right)^{*} & (0 \leq m \leq n-3, q \in\{n-1, n\}) ; \\
u_{i, q} & =e_{i, q}{ }^{*} & (q \in\{n-1, n\}) ; \\
v_{i, q} & =\left(\gamma_{i+1, q} \nu_{i+1,1} \beta_{i, q}\right)^{*} & & (q-1, n\}) .
\end{array}
$$

Now, we pick some elements of the algebra $\mathrm{HH}^{*}(R)$.

a) Suppose $s=2 m+l(2 n-3), 0 \leq m \leq n-2, r|m+l(n-1), 2| m+l n$, and either char $k=2$ or $2 \mid l$. We define an $s$-cocycle $f_{s} \in \operatorname{Ker} \delta^{s}$ by

$$
f_{s}=\sum_{i=1}^{r}\left(\sum_{j=1}^{n-2-m} w_{i, m, j}+u_{i, n-1}+u_{i, n}\right) .
$$

b) Suppose $s=2 m+1+l(2 n-3), 0 \leq m \leq n-3, r \mid m+l(n-1), 2 \nmid m+l n$, and either char $k=2$ or $2 \nmid l$. We define an $s$-cocycle $g_{s} \in \operatorname{Ker} \delta^{s}$ by

$$
g_{s}=\sum_{i=1}^{r}\left(\sum_{j=n-1-m}^{n-2} t_{i, m, j}+u_{i, m, n-1}+v_{i, m, n-1}\right) .
$$

c) Suppose $s=(l+1)(2 n-3)-1, r \mid(l+1)(n-1)-1$, and $2 \nmid(l+1) n$. We define an $s$-cocycle $h_{s} \in \operatorname{Ker} \delta^{s}$ by

$$
h_{s}=\sum_{i=1}^{r} \sum_{j=1}^{n-2}(-1)^{j} w_{i, 0, j}
$$

d) Suppose $s=(l+1)(2 n-3)-1, r|(l+1)(n-1)-1,2| n$, and either char $k=2$ or $2 \mid l$. We define an $s$-cocycle $p_{s} \in \operatorname{Ker} \delta^{s}$ by

$$
p_{s}=\sum_{i=1}^{r}\left(\sum_{j=1}^{n-2}(-1)^{j} w_{i, 0, j}+u_{i, n-1}\right) .
$$

Using the description of the homomorphisms $d_{s}$ in [9], it is easy to verify that $f_{s}, g_{s}$, $h_{s}$, and $p_{s}$ defined above are indeed cocycles.

Remark 2. In the rest of the paper, we suppose that $s=2 m+l(2 n-3)$ or $s=2 m+$ $1+l(2 n-3)$, where $0 \leq s-l(2 n-3) \leq 2 n-4$. The additional conditions imposed on $s$ will show each time what version indeed occurs.

Proposition 1. 1) Assume that the element $f_{s}$ is defined for some s. Then $T_{t}\left(f_{s}\right)$ : $Q_{t+s} \rightarrow Q_{t}$ can be defined on the direct summands of $Q_{t+s}$ in the following way:

1.1) if $t=2 q, 0 \leq q \leq n-2-m$, then

$$
\begin{array}{lr}
\left.T_{t}\left(f_{s}\right)\right|_{P_{[i+q, j+m+q][i, j]}}=e_{i+q, j+m+q} \otimes \alpha_{i, j+m-1} \ldots \alpha_{i, j} & (1 \leq j \leq n-2-m-q), \\
\left.T_{t}\left(f_{s}\right)\right|_{P_{[i+q, j+m+q-(n-2)][i, j]}}=0 & (n-1-m-q \leq j \leq n-2-q),
\end{array}
$$




$$
\begin{array}{rr}
\left.T_{t}\left(f_{s}\right)\right|_{P_{[i+q, j+m+q-(n-2)][i, j]}}=\alpha_{i+q, j+m+q-(n-1)} \ldots \alpha_{i+q, j+q-(n-2)} \otimes e_{i, j} \\
(n-1-q \leq j \leq n-2), \\
\left.T_{t}\left(f_{s}\right)\right|_{P_{\left[i+q, \phi^{q}(j)\right][i, j]}=e_{i+q, \phi^{q}(j)} \otimes e_{i, j}}(j \in\{n-1, n\}) ;
\end{array}
$$

1.2) if $t=2 q+1,0 \leq q \leq n-3-m$, then

$$
\begin{array}{r}
\left.T_{t}\left(f_{s}\right)\right|_{P_{[i+q, j+m+q+1][i, j]}}=e_{i+q, j+m+q+1} \otimes \alpha_{i, j+m-1} \ldots \alpha_{i, j} \quad(1 \leq j \leq n-3-m-q), \\
\left.T_{t}\left(f_{s}\right)\right|_{P_{\left[i+q, \phi^{q}(j)\right][i, n-2-m-q]}}=e_{i+q, \phi^{q}(j)} \otimes \alpha_{i, n-3-q} \ldots \alpha_{i, n-2-m-q} \quad(j \in\{n-1, n\}), \\
\left.T_{t}\left(f_{s}\right)\right|_{P_{[i+q+1, j+m+q-(n-2)][i, j]}}=\mu_{i+q+1, j+m+q-(n-1)} \beta_{i+q, \phi^{q}(n)} \otimes \alpha_{i, n-3-q} \ldots \alpha_{i, j} \\
-\mu_{i+q+1, j+m+q-(n-1)} \beta_{i+q, \phi^{q}(n-1)} \otimes \alpha_{i, n-3-q} \ldots \alpha_{i, j} \\
(n-1-m-q \leq j \leq n-2-q),
\end{array}
$$

$\left.T_{t}\left(f_{s}\right)\right|_{P_{[i+q+1, j+m+q-(n-2)][i, j]}}=\alpha_{i+q+1, j+m+q-(n-1)} \ldots \alpha_{i+q+1, j+q-(n-2)} \otimes e_{i, j}$

$$
(n-1-q \leq j \leq n-2),
$$

$$
\begin{aligned}
\left.T_{t}\left(f_{s}\right)\right|_{P_{[i+q+1, m+q+1][i, j]}}= & \sum_{z=1}^{m} \alpha_{i+q+1, m+q} \ldots \alpha_{i+q+1, z+q+1} \otimes \mu_{i+1, z-1} \beta_{i, j} \\
& \quad+\alpha_{i+q+1, m+q} \ldots \alpha_{i+q+1, q+1} \otimes e_{i, j} \quad(j \in\{n-1, n\}) ;
\end{aligned}
$$

1.3) if $t=2 n-3-2 m+2 q, 0 \leq q \leq m-1$, then

$$
\begin{aligned}
& \left.T_{t}\left(f_{s}\right)\right|_{P_{[i+q+n-1-m, j+q][i, j]}} \\
& =(-1)^{q}\left(\mu_{i+q+n-1-m, j+q-1} \beta_{i+q+n-2-m, \phi^{q+n-m}(n)} \otimes \alpha_{i, m-q-1} \ldots \alpha_{i, j}\right. \\
& \left.-\mu_{i+q+n-1-m, j+q-1} \beta_{i+q+n-2-m, \phi^{q+n-m}(n-1)} \otimes \alpha_{i, m-q-1} \ldots \alpha_{i, j}\right) \\
& (1 \leq j \leq m-q), \\
& \left.T_{t}\left(f_{s}\right)\right|_{P_{[i+q+n-1-m, j+q][i, j]}}=(-1)^{q} \alpha_{i+q+n-1-m, j+q-1} \ldots \alpha_{i+q+n-1-m, j+q-m} \otimes e_{i, j} \\
& (m-q+1 \leq j \leq n-2-q) \text {, } \\
& \left.T_{t}\left(f_{s}\right)\right|_{P_{[i+q+n-1-m, j+q-(n-2)][i, j]}}=0 \quad(n-1-q \leq j \leq n-2) \text {, } \\
& \left.T_{t}\left(f_{S}\right)\right|_{\left.P_{[i+q+n-1-m, \phi} q+n-m(j)\right][i, j]} \\
& =(-1)^{j+q-n}\left(e_{i+q+n-1-m, \phi^{q+n-m}(j)} \otimes \mu_{i+1, m-q-1} \beta_{i, j}\right. \\
& +\sum_{z=1}^{m-q-1} \gamma_{i+q+n-1-m, \phi^{q+n-m}(j)} \nu_{i+q+n-1-m, z+q+n-1-m} \otimes \mu_{i+1, z-1} \beta_{i, j} \\
& \left.+\gamma_{i+q+n-1-m, \phi^{q+n-m}(j)} \nu_{i+q+n-1-m, q+n-1-m} \otimes e_{i, j}\right) \quad(j \in\{n-1, n\}) ;
\end{aligned}
$$

1.4) if $t=2 n-2-2 m+2 q, 0 \leq q \leq m-1$, then

$$
\begin{array}{rr}
\left.T_{t}\left(f_{s}\right)\right|_{P_{[i+q+n-1-m, j+q+1][i, j]}}=0 & (1 \leq j \leq m-q-1), \\
\left.T_{t}\left(f_{s}\right)\right|_{P_{[i+q+n-1-m, j+q+1][i, j]}}=(-1)^{q} \alpha_{i+q+n-1-m, j+q} \ldots \alpha_{i+q+n-1-m, j+q+1-m} \otimes e_{i, j} \\
& (m-q \leq j \leq n-3-q),
\end{array}
$$

$$
\begin{aligned}
& \left.T_{t}\left(f_{s}\right)\right|_{P_{\left[i+q+n-1-m, \phi^{q+n-m}(j)\right][i, n-2-q]}} \\
& =(-1)^{q}\left(-\sum_{z=n-2-q}^{n-2} \gamma_{i+q+n-1-m, \phi^{q+n-m}(j)} \nu_{i+q+n-1-m, z+q-m+1} \otimes \alpha_{i, z-1} \ldots \alpha_{i, n-2-q}\right. \\
& \left.\quad+(-1)^{j-n} e_{i+q+n-1-m, \phi^{q+n-m}(j)} \otimes \gamma_{i, \phi(j)} \nu_{i, n-2-q}\right) \quad(j \in\{n-1, n\}),
\end{aligned}
$$




$$
\begin{aligned}
& \left.T_{t}\left(f_{s}\right)\right|_{P_{[i+q+n-m, j+q-(n-2)][i, j]}} \\
& =(-1)^{q}\left(\mu_{i+q+n-m, j+q-n} \beta_{i+q+n-1-m, \phi^{q+n-m}(n)} \otimes \gamma_{i, n-1} \nu_{i, j}\right. \\
& \left.+\mu_{i+q+n-m, j+q-n} \beta_{i+q+n-1-m, \phi^{q+n-m}(n-1)} \otimes \gamma_{i, n} \nu_{i, j}\right) \\
& \quad(n-1-q \leq j \leq n-2), \\
& \left.T_{t}\left(f_{s}\right)\right|_{P_{[i+q+n-m, q+1][i, j]}=(-1)^{j+q-(n-1)} \mu_{i+q+n-m, q} \beta_{i+q+n-1-m, \phi^{q+n-m-1}(j)} \otimes e_{i, j}} \quad(j \in\{n-1, n\}) ;
\end{aligned}
$$

1.5) if $t=u+v(2 n-3)$ with $0 \leq u \leq 2 n-4$ and $v \geq 1$, then $T_{t}\left(f_{s}\right)=\sigma^{v}\left(T_{u}\left(f_{s}\right)\right)$, where $T_{u}\left(f_{s}\right)$ is described by the formulas in 1.1)-1.4).

2) Assume that the element $g_{s}$ is defined. Then $T_{t}\left(g_{s}\right): Q_{t+s} \rightarrow Q_{t}$ can be defined on the direct summands of $Q_{t+s}$ as follows:

2.1) if $t=2 q, 0 \leq q \leq n-3-m$, then

$$
\begin{aligned}
& \left.T_{t}\left(g_{s}\right)\right|_{P_{[i+q, j+m+q+1][i, j]}}=0 \\
& \left.T_{t}\left(g_{s}\right)\right|_{\left.P_{[i+q, \phi} q(n)\right][i, n-2-m-q]}=0, \\
& \left.T_{t}\left(g_{s}\right)\right|_{\left.P_{[i+q, \phi} q(n-1)\right][i, n-2-m-q]}=(-1)^{q} e_{i+q, \phi^{q}(n-1)} \otimes \gamma_{i, n-1} \nu_{i, n-2-m-q}, \\
& \left.T_{t}\left(g_{s}\right)\right|_{P_{[i+q+1, m+q+1][i, n]}}=0, \\
& \left.T_{t}\left(g_{s}\right)\right|_{P_{[i+q+1, m+q+1][i, n-1]}} \\
& \quad=(-1)^{q}\left(\sum_{z=n-1-q}^{n-2} \alpha_{i+q+1, m+q} \ldots \alpha_{i+q+1, z+q-(n-2)} \otimes \mu_{i+1, z-1} \beta_{i, n-1}\right. \\
& \left.\quad+\mu_{i+q+1, m+q} \beta_{i+q, \phi^{q}(n-1)} \otimes e_{i, n-1}\right),
\end{aligned}
$$

$\left.T_{t}\left(g_{s}\right)\right|_{P_{[i+q+1, j+m+q-(n-2)][i, j]}}$

$$
\begin{array}{r}
=(-1)^{q}\left(\sum_{z=n-1-q}^{j} \alpha_{i+q+1, j+m+q-(n-1)} \ldots \alpha_{i+q+1, z+q-(n-2)} \otimes \mu_{i+1, z-1} \tau_{i} \nu_{i, j}\right. \\
\left.+\mu_{i+q+1, j+m+q-(n-1)} \beta_{i+q, \phi^{q}(n-1)} \otimes \gamma_{i, n-1} \nu_{i, j}\right) \\
(n-1-m-q \leq j \leq n-2) ;
\end{array}
$$

2.2) if $t=2 q+1,0 \leq q \leq n-3-m$, then

$$
\begin{aligned}
& \left.T_{t}\left(g_{s}\right)\right|_{P_{[i+q+1, j+m+q+1][i, j]}} \\
& \left.\begin{array}{r}
=(-1)^{q+1}\left(\sum_{z=1}^{j} \alpha_{i+q+1, j+m+q} \ldots \alpha_{i+q+1, z+q+1} \otimes \mu_{i+1, z-1} \tau_{i} \nu_{i, j}\right. \\
+\alpha_{i+q+1, j+m+q} \ldots \alpha_{i+q+1, q+1} \otimes \gamma_{i, n-1} \nu_{i, j}
\end{array}\right) \\
& (1 \leq j \leq n-3-m-q) \text {, } \\
& \left.T_{t}\left(g_{s}\right)\right|_{P_{[i+q+1, j+m+q-(n-3)][i, j]}}=0 \quad(n-2-m-q \leq j \leq n-2), \\
& \left.T_{t}\left(g_{s}\right)\right|_{P_{\left[i+q+1, \phi^{q}(n)\right][i, n]}}=0 \text {, } \\
& \left.T_{t}\left(g_{s}\right)\right|_{P_{\left[i+q+1, \phi^{q}(n-1)\right][i, n-1]}}=(-1)^{q}\left(e_{i+q+1, \phi^{q}(n-1)} \otimes \mu_{i+1, n-3-q} \beta_{i, n-1}\right. \\
& +\sum_{z=1}^{n-3-q} \gamma_{i+q+1, \phi^{q}(n-1)} \nu_{i+q+1, z+q+1} \otimes \mu_{i+1, z-1} \beta_{i, n-1} \\
& \left.+\gamma_{i+q+1, \phi^{q}(n-1)} \nu_{i+q+1, q+1} \otimes e_{i, n-1}\right) \text {; }
\end{aligned}
$$


2.3) if $t=2 n-4-2 m$, then

$$
\begin{aligned}
& \begin{aligned}
&\left.T_{t}\left(g_{s}\right)\right|_{P_{[i+n-1-m, j][i, j]}}=-\sum_{z=m+1}^{j} \alpha_{i+n-1-m, j-1} \ldots \alpha_{i+n-1-m, z-m} \otimes \mu_{i+1, z-1} \tau_{i} \nu_{i, j} \\
&-\mu_{i+n-1-m, j-1} \beta_{i+n-2-m, \phi^{n-1-m}(n)} \otimes \gamma_{i, n-1} \nu_{i, j} \\
& \quad(1 \leq j \leq n-2),
\end{aligned} \\
& \left.T_{t}\left(g_{s}\right)\right|_{P_{\left[i+n-1-m, \phi^{n-1-m}(n)\right][i, n]}}=0, \\
& \left.T_{t}\left(g_{s}\right)\right|_{P_{\left[i+n-1-m, \phi^{n-1-m}(n-1)\right][i, n-1]}} \\
& =-\sum_{z=m+1}^{n-2} \gamma_{i+n-1-m, \phi^{n-1-m}(n-1)} \nu_{i+n-1-m, z-m} \otimes \mu_{i+1, z-1} \beta_{i, n-1} ;
\end{aligned}
$$

2.4) if $t=2 n-4-2 m+2 q, 1 \leq q \leq m$, then

$\left.T_{t}\left(g_{s}\right)\right|_{P}$

$$
\begin{array}{r}
=-\sum_{z=m+1}^{j} \alpha_{i+q+n-1-m, j+q-1} \ldots \alpha_{i+q+n-1-m, z+q-m} \otimes \mu_{i+1, z-1} \tau_{i} \nu_{i, j} \\
(1 \leq j \leq n-2-q),
\end{array}
$$

$\left.T_{t}\left(g_{s}\right)\right|_{P_{[i+q+n-1-m, \phi} q+n-1-m_{(n)][i, n]}}=0$,

$\left.T_{t}\left(g_{s}\right)\right|_{P_{\left[i+q+n-1-m, \phi^{q+n-1-m}(n-1)\right][i, n-1]}}$

$=\sum_{z=n-1-q}^{m} \gamma_{i+q+n-1-m, \phi^{q+n-1-m}(n-1)} \nu_{i+q+n-1-m, z+q-m} \otimes \mu_{i+1, z-1} \beta_{i, n-1}$

$-\sum_{z=m+1}^{n-2-q} \gamma_{i+q+n-1-m, \phi^{q+n-1-m}(n-1)} \nu_{i+q+n-1-m, z+q-m} \otimes \mu_{i+1, z-1} \beta_{i, n-1}$,

$\left.T_{t}\left(g_{s}\right)\right|_{P_{[i+q+n-1-m, 1][i, n-1-q]}}=e_{i+q+n-1-m, 1} \otimes \mu_{i+1, m-q} \tau_{i} \nu_{i, n-1-q}$

$$
\begin{aligned}
& -\beta_{i+q+n-1-m, \phi^{q+n-1-m}(n-1)} \otimes \gamma_{i, n} \nu_{i, n-1-q} \\
& -\sum_{z=n-1-q}^{n-2} \tau_{i+q+n-2-m}^{+} \beta_{i+q+n-1-m, \phi^{q+n-1-m}(n)} \otimes \gamma_{i, n-1} \nu_{i, n-1-q}
\end{aligned}
$$

$\left.T_{t}\left(g_{s}\right)\right|_{P_{[i+q+n-1-m, j+q-(n-2)][i, j]}}=e_{i+q+n-1-m, j+q-(n-2)} \otimes \mu_{i+1, j+m-(n-1)} \tau_{i} \nu_{i, j}$

$$
(n-q \leq j \leq n-2)
$$

2.5) if $t=2 n-3-2 m+2 q, 0 \leq q \leq m-1$, then

$$
\begin{aligned}
& \left.T_{t}\left(g_{s}\right)\right|_{P_{[i+q+n-1-m, j+q+1][i, j]}}=0 \quad(1 \leq j \leq n-3-q), \\
& \left.T_{t}\left(g_{s}\right)\right|_{\left.P_{[i+q+n-1-m, \phi} q+n-1-m(n-1)\right][i, n-2-q]}=0 \text {, } \\
& \left.T_{t}\left(g_{s}\right)\right|_{P_{[i+q+n-1-m, \phi} q+n-1-m_{(n)][i, n-2-q]}} \\
& =-\gamma_{i+q+n-1-m, \phi^{q+n-1-m}(n)} \nu_{i+q+n-1-m, n-2-m} \otimes e_{i, n-2-q}, \\
& \left.T_{t}\left(g_{s}\right)\right|_{P_{[i+q+n-m, q+1][i, n]}}=0 \text {, } \\
& \left.T_{t}\left(g_{s}\right)\right|_{P_{[i+q+n-m, q+1][i, n-1]}}=e_{i+q+n-m, q+1} \otimes \mu_{i+1, m} \beta_{i, n-1},
\end{aligned}
$$




$$
\begin{array}{r}
\left.T_{t}\left(g_{s}\right)\right|_{P_{[i+q+n-m, j+q-(n-2)][i, j]}}=e_{i+q+n-m, j+q-(n-2)} \otimes \mu_{i+1, j+m-(n-1)} \tau_{i} \nu_{i, j} \\
(n-1-q \leq j \leq n-2) ;
\end{array}
$$

2.6) if $t=u+v(2 n-3)$ with $0 \leq u \leq 2 n-4$ and $v \geq 1$, then $T_{t}\left(g_{s}\right)=\sigma^{v}\left(T_{u}\left(g_{s}\right)\right)$, where $T_{u}\left(g_{s}\right)$ is described by the formulas in 2.1)-2.5).

3) Assume that the element $h_{s}$ is defined. Then $T_{t}\left(h_{s}\right): Q_{t+s} \rightarrow Q_{t}$ can be defined on the direct summands of $Q_{t+s}$ as follows:

3.1) if $t=0$, then

$$
\begin{array}{ll}
\left.T_{t}\left(h_{s}\right)\right|_{P_{[i, j][i, j]}}=(-1)^{j} e_{i, j} \otimes e_{i, j} & (1 \leq j \leq n-2), \\
\left.T_{t}\left(h_{s}\right)\right|_{P_{[i, \phi(j)][i, j]}}=0 & (j \in\{n-1, n\}) ;
\end{array}
$$

3.2) if $t=4 q, 1 \leq q \leq(n-3) / 2$, then

$$
\begin{aligned}
& \left.T_{t}\left(h_{s}\right)\right|_{P_{[i+2 q, j+2 q][i, j]}}=(-1)^{j+1} e_{i+2 q, j+2 q} \otimes e_{i, j} \\
& \left.T_{t}\left(h_{s}\right)\right|_{P_{[i+2 q, n-1][i, n-1-2 q]}}=0, \\
& \left.T_{t}\left(h_{s}\right)\right|_{P_{[i+2 q, n][i, n-1-2 q]}}=\sum_{z=\frac{n-1}{2}-q}^{\frac{n-3}{2}} \gamma_{i+2 q, n} \nu_{i+2 q, 2 z+2 q-(n-2)} \otimes \alpha_{i, 2 z-1} \ldots \alpha_{i, n-1-2 q},
\end{aligned}
$$

$\left.T_{t}\left(h_{s}\right)\right|_{P_{[i+2 q+1,2 j+2 q-(n-1)][i, 2 j]}}$

$$
\begin{aligned}
& =\sum_{z=j}^{\frac{n-3}{2}} \mu_{i+2 q+1,2 j+2 q-n} \tau_{i+2 q} \nu_{i+2 q, 2 z+2 q-(n-2)} \otimes \alpha_{i, 2 z-1} \ldots \alpha_{i, 2 j} \\
& \quad-\sum_{z=\frac{n-1}{2}-q}^{j-1} \alpha_{i+2 q+1,2 j+2 q-n} \ldots \alpha_{i+2 q+1,2 z+2 q-(n-3)} \otimes \mu_{i+1,2 z} \tau_{i} \nu_{i, 2 j} \\
& \quad\left(\frac{n+1}{2}-q \leq j \leq \frac{n-3}{2}\right),
\end{aligned}
$$

$\left.T_{t}\left(h_{s}\right)\right|_{P_{[i+2 q+1,2 j+2 q-(n-2)][i, 2 j+1]}}$

$$
\begin{aligned}
= & -\sum_{z=j}^{\frac{n-3}{2}} \begin{array}{r}
\mu_{i+2 q+1,2 j+2 q-(n-1)} \tau_{i+2 q} \nu_{i+2 q, 2 z+2 q-(n-3)} \otimes \alpha_{i, 2 z} \ldots \alpha_{i, 2 j+1} \\
\quad+\mu_{i+2 q+1,2 j+2 q-(n-1)} \beta_{i+2 q, n-1} \otimes \gamma_{i, n-1} \nu_{i, 2 j+1}
\end{array} \\
& \quad+\sum^{j} \begin{array}{l}
\quad-\mu_{i+2 q+1,2 j+2 q-(n-1)} \beta_{i+2 q, n} \otimes \gamma_{i, n} \nu_{i, 2 j+1} \\
\alpha_{i+2 q+1,2 j+2 q-(n-1)} \ldots \alpha_{i+2 q+1,2 z+2 q-(n-2)} \otimes \mu_{i+1,2 z-1} \tau_{i} \nu_{i, 2 j+1}
\end{array} \\
& \quad \quad\left(\frac{n-1}{2}-q \leq j \leq \frac{n-3}{2}\right),
\end{aligned}
$$

$\left.T_{t}\left(h_{s}\right)\right|_{P_{[i+2 q+1,2 q][i, n]}}=0$,

$\left.T_{t}\left(h_{s}\right)\right|_{P_{[i+2 q+1,2 q][i, n-1]}}=-\sum_{z=\frac{n-1}{2}-q}^{\frac{n-3}{2}} \alpha_{i+2 q+1,2 q-1} \ldots \alpha_{i+2 q+1,2 z+2 q-(n-3)} \otimes \mu_{i+1,2 z} \beta_{i, n-1} ;$

3.3) if $t=4 q+2,0 \leq q \leq(n-3) / 2$, then

$$
\begin{aligned}
\left.T_{t}\left(h_{s}\right)\right|_{P_{[i+2 q+1, j+2 q+1][i, j]}}= & (-1)^{j} e_{i+2 q+1, j+2 q+1} \otimes e_{i, j} \quad(1 \leq j \leq n-3-2 q), \\
\left.T_{t}\left(h_{s}\right)\right|_{P_{[i+2 q+1, n][i, n-2-2 q]}}= & e_{i+2 q+1, n} \otimes \gamma_{i, n-1} \nu_{i, n-2-2 q}, \\
\left.T_{t}\left(h_{s}\right)\right|_{P_{[i+2 q+1, n-1][i, n-2-2 q]}} & =\sum_{z=\frac{n-3}{2}-q}^{\frac{n-3}{2}} \gamma_{i+2 q+1, n-1} \nu_{i+2 q+1,2 z+2 q-(n-4)} \otimes \alpha_{i, 2 z} \ldots \alpha_{i, n-2-2 q}+e_{i+2 q+1, n-1} \otimes \gamma_{i, n} \nu_{i, n-2-2 q},
\end{aligned}
$$




$$
\begin{aligned}
& \left.T_{t}\left(h_{s}\right)\right|_{P_{[i+2 q+2,2 j+2 q-(n-2)][i, 2 j]}} \\
& =-\sum_{z=j}^{\frac{n-3}{2}} \mu_{i+2 q+2,2 j+2 q-(n-1)} \tau_{i+2 q+1} \nu_{i+2 q+1,2 z+2 q-(n-3)} \otimes \alpha_{i, 2 z-1} \ldots \alpha_{i, 2 j} \\
& \quad+\sum_{z=\frac{n-3}{2}-q} \alpha_{i+2 q+2,2 j+2 q-(n-1)} \ldots \alpha_{i+2 q+2,2 z+2 q-(n-4)} \otimes \mu_{i+1,2 z} \tau_{i} \nu_{i, 2 j} \\
& \quad\left(\frac{n-1}{2}-q \leq j \leq \frac{n-3}{2}\right),
\end{aligned}
$$

$\left.T_{t}\left(h_{s}\right)\right|_{P_{[i+2 q+2,2 j+2 q-(n-3)][i, 2 j+1]}}$

$$
\begin{aligned}
& =\sum_{z=j}^{\frac{n-3}{2}} \mu_{i+2 q+2,2 j+2 q-(n-2)} \tau_{i+2 q+1} \nu_{i+2 q+1,2 z+2 q-(n-4)} \otimes \alpha_{i, 2 z} \ldots \alpha_{i, 2 j+1} \\
& -\mu_{i+2 q+2,2 j+2 q-(n-2)} \beta_{i+2 q+1, n} \otimes \gamma_{i, n-1} \nu_{i, 2 j+1} \\
& +\mu_{i+2 q+2,2 j+2 q-(n-2)} \beta_{i+2 q+1, n-1} \otimes \gamma_{i, n} \nu_{i, 2 j+1}
\end{aligned}
$$

$\left.T_{t}\left(h_{s}\right)\right|_{P_{[i+2 q+2,2 q+1][i, n]}}=0$,

$\left.T_{t}\left(h_{s}\right)\right|_{P_{[i+2 q+2,2 q+1][i, n-1]}}=\sum_{z=\frac{n-3}{2}-q}^{\frac{n-3}{2}} \alpha_{i+2 q+2,2 q} \ldots \alpha_{i+2 q+2,2 z+2 q-(n-4)} \otimes \mu_{i+1,2 z} \beta_{i, n-1} ;$

3.4) if $t=4 q+1,0 \leq q \leq(n-3) / 2$, then

$$
\begin{aligned}
& \left.T_{t}\left(h_{s}\right)\right|_{P_{[i+2 q+1,2 j+2 q][i, 2 j]}} \\
& =-\sum_{z=j}^{\frac{n-3}{2}-q} \mu_{i+2 q+1,2 j+2 q-1} \tau_{i+2 q} \nu_{i+2 q, 2 z+2 q+1} \otimes \alpha_{i, 2 z-1} \ldots \alpha_{i, 2 j} \\
& \quad-\sum_{z=0}^{j-1} \alpha_{i+2 q+1,2 j+2 q-1} \ldots \alpha_{i+2 q+1,2 z+2 q+2} \otimes \mu_{i+1,2 z} \tau_{i} \nu_{i, 2 j} \quad\left(1 \leq j \leq \frac{n-3}{2}-q\right),
\end{aligned}
$$

$\left.T_{t}\left(h_{s}\right)\right|_{P_{[i+2 q+1,2 j+2 q+1][i, 2 j+1]}}$

$$
\begin{aligned}
& =\sum_{z=j}^{\frac{n-5}{2}-q} \begin{array}{r}
\mu_{i+2 q+1,2 j+2 q} \tau_{i+2 q} \nu_{i+2 q, 2 z+2 q+2} \otimes \alpha_{i, 2 z} \ldots \alpha_{i, 2 j+1} \\
+ \\
+\mu_{i+2 q+1,2 j+2 q} \beta_{i+2 q, n-1} \otimes \alpha_{i, n-3-2 q} \ldots \alpha_{i, 2 j+1} \\
+\alpha_{i+2 q+1,2 j+2 q} \ldots \alpha_{i+2 q+1,2 q+1} \otimes \gamma_{i, n-1} \nu_{i, 2 j+1}
\end{array} \\
& \quad+\sum_{z=1}^{j} \alpha_{i+2 q+1,2 j+2 q} \ldots \alpha_{i+2 q+1,2 z+2 q+1} \otimes \mu_{i+1,2 z-1} \tau_{i} \nu_{i, 2 j+1} \\
& \quad\left(0 \leq j \leq \frac{n-3}{2}-q\right),
\end{aligned}
$$

$\left.T_{t}\left(h_{s}\right)\right|_{P_{[i+2 q+1, \phi(j)][i, j]}}=\sum_{z=0}^{\frac{n-5}{2}-q} \gamma_{i+2 q+1, \phi(j)} \nu_{i+2 q+1,2 z+2 q+2} \otimes \mu_{i+1,2 z} \beta_{i, j}$

$$
+e_{i+2 q+1, \phi(j)} \otimes \mu_{i+1, n-3-2 q} \beta_{i, j} \quad(j \in\{n-1, n\}),
$$

$\left.T_{t}\left(h_{s}\right)\right|_{P_{[i+2 q+1, j+2 q-(n-2)][i, j]}}=(-1)^{j+1} e_{i+2 q+1, j+2 q-(n-2)} \otimes e_{i, j}$

$$
(n-1-2 q \leq j \leq n-2) ;
$$


3.5) if $t=4 q+3,0 \leq q \leq(n-5) / 2$, then

$$
\begin{aligned}
& \left.T_{t}\left(h_{s}\right)\right|_{P_{[i+2 q+2,2 j+2 q+1][i, 2 j]}} \\
& =\sum_{z=j}^{\frac{n-5}{2}-q} \mu_{i+2 q+2,2 j+2 q} \tau_{i+2 q+1} \nu_{i+2 q+1,2 z+2 q+2} \otimes \alpha_{i, 2 z-1} \ldots \alpha_{i, 2 j} \\
& \quad+\sum_{z=0}^{j-1} \alpha_{i+2 q+2,2 j+2 q} \ldots \alpha_{i+2 q+2,2 z+2 q+3} \otimes \mu_{i+1,2 z} \tau_{i} \nu_{i, 2 j} \quad\left(1 \leq j \leq \frac{n-3}{2}-q\right),
\end{aligned}
$$

$\left.T_{t}\left(h_{s}\right)\right|_{P_{[i+2 q+2,2 j+2 q+2][i, 2 j+1]}}$

$$
\begin{aligned}
&=-\sum_{z=j}^{\frac{n-5}{2}-q} \mu_{i+2 q+2,2 j+2 q+1} \tau_{i+2 q+1} \nu_{i+2 q+1,2 z+2 q+3} \otimes \alpha_{i, 2 z} \ldots \alpha_{i, 2 j+1} \\
&- \alpha_{i+2 q+2,2 j+2 q+1} \ldots \alpha_{i+2 q+2,2 q+2} \otimes \gamma_{i, n-1} \nu_{i, 2 j+1} \\
&-\sum_{z=1}^{j} \alpha_{i+2 q+2,2 j+2 q+1} \ldots \alpha_{i+2 q+2,2 z+2 q+2} \otimes \mu_{i+1,2 z-1} \tau_{i} \nu_{i, 2 j+1} \\
& \quad(0 \leq j \leq
\end{aligned}
$$$$
\left(0 \leq j \leq \frac{n-5}{2}-q\right),
$$

$\left.T_{t}\left(h_{s}\right)\right|_{P_{[i+2 q+2, j][i, j]}}=-\sum_{z=0}^{\frac{n-5}{2}-q} \gamma_{i+2 q+2, j} \nu_{i+2 q+2,2 z+2 q+3} \otimes \mu_{i+1,2 z} \beta_{i, j}$

$$
(j \in\{n-1, n\}),
$$

$\left.T_{t}\left(h_{s}\right)\right|_{P_{[i+2 q+2, j+2 q-(n-3)][i, j]}}=(-1)^{j} e_{i+2 q+2, j+2 q-(n-3)} \otimes e_{i, j}$

$$
(n-2-2 q \leq j \leq n-2)
$$

3.6) if $t=u+v(2 n-3)$ with $0 \leq u \leq 2 n-4$ and $v \geq 1$, then $T_{t}\left(h_{s}\right)=\sigma^{v}\left(T_{u}\left(h_{s}\right)\right)$, where $T_{u}\left(h_{s}\right)$ is described by the formulas in 3.1)-3.5).

4) Assume that the element $p_{s}$ is defined. Then $T_{t}\left(p_{s}\right): Q_{t+s} \rightarrow Q_{t}$ can be defined on the direct summands of $Q_{t+s}$ as follows:

4.1) if $t=0$, then

$$
\begin{aligned}
& \left.T_{t}\left(p_{s}\right)\right|_{P_{[i, j][i, j]}}=(-1)^{j} e_{i, j} \otimes e_{i, j} \quad \quad(1 \leq j \leq n-2), \\
& \left.T_{t}\left(p_{s}\right)\right|_{P_{[i, n][i, n]}}=0, \\
& \left.T_{t}\left(p_{s}\right)\right|_{P_{[i, n-1][i, n-1]}}=e_{i, n-1} \otimes e_{i, n-1} ;
\end{aligned}
$$

4.2) if $t=4 q, 1 \leq q \leq(n-2) / 2$, then

$$
\begin{array}{ll}
\left.T_{t}\left(p_{s}\right)\right|_{P_{[i+2 q, j+2 q][i, j]}}=(-1)^{j+1} e_{i+2 q, j+2 q} \otimes e_{i, j} & (1 \leq j \leq n-2-2 q), \\
\left.T_{t}\left(p_{s}\right)\right|_{P_{[i+2 q, n][i, n-1-2 q]}}=0, & 0 \\
\left.T_{t}\left(p_{s}\right)\right|_{P_{[i+2 q, n-1][i, n-1-2 q]}}=\sum_{z=\frac{n-2}{2}-q}^{\frac{n-4}{2}} \gamma_{i+2 q, n-1} \nu_{i+2 q, 2 z+2 q-(n-3)} \otimes \alpha_{i, 2 z} \ldots \alpha_{i, n-1-2 q}
\end{array}
$$


$\left.T_{t}\left(p_{s}\right)\right|_{P_{[i+2 q+1,2 j+2 q-(n-1)][i, 2 j]}}$

$$
\begin{aligned}
&=-\sum_{z=j}^{\frac{n-2}{2}} \mu_{i+2 q+1,2 j+2 q-n} \tau_{i+2 q} \nu_{i+2 q, 2 z+2 q-(n-2)} \otimes \alpha_{i, 2 z-1} \ldots \alpha_{i, 2 j} \\
&+\sum_{z=\frac{n-2}{2}-q}^{j-1} \alpha_{i+2 q+1,2 j+2 q-n} \ldots \alpha_{i+2 q+1,2 z+2 q-(n-3)} \otimes \mu_{i+1,2 z} \tau_{i} \nu_{i, 2 j} \\
& \quad\left(\frac{n}{2}-q \leq j \leq \frac{n-2}{2}\right),
\end{aligned}
$$

$\left.T_{t}\left(p_{s}\right)\right|_{P_{[i+2 q+1,2 j+2 q-(n-2)][i, 2 j+1]}}$

$$
\begin{gathered}
=\sum_{z=j}^{\frac{n-4}{2}} \mu_{i+2 q+1,2 j+2 q-(n-1)} \tau_{i+2 q} \nu_{i+2 q, 2 z+2 q-(n-3)} \otimes \alpha_{i, 2 z} \ldots \alpha_{i, 2 j+1} \\
\quad-\sum_{i+2 q+1,2 j+2 q-(n-1)} \beta_{i+2 q, n-1} \otimes \gamma_{i, n-1} \nu_{i, 2 j+1} \\
\quad \sum_{z=\frac{n}{2}-q}^{j} \alpha_{i+2 q+1,2 j+2 q-(n-1)} \ldots \alpha_{i+2 q+1,2 z+2 q-(n-2)} \otimes \mu_{i+1,2 z-1} \tau_{i} \nu_{i, 2 j+1} \\
\quad\left(\frac{n}{2}-q \leq j \leq \frac{n-4}{2}\right),
\end{gathered}
$$

$\left.T_{t}\left(p_{s}\right)\right|_{P_{[i+2 q+1,2 q][i, n]}}=0$,

$\left.T_{t}\left(p_{s}\right)\right|_{P_{[i+2 q+1,2 q][i, n-1]}}=-\mu_{i+2 q+1,2 q-1} \beta_{i+2 q, n-1} \otimes e_{i, n-1}$

$$
-\sum_{z=\frac{n}{2}-q}^{\frac{n-2}{2}} \alpha_{i+2 q+1,2 q-1} \ldots \alpha_{i+2 q+1,2 z+2 q-(n-2)} \otimes \mu_{i+1,2 z-1} \beta_{i, n-1}
$$

4.3) if $t=4 q+2,0 \leq q \leq(n-4) / 2$, then

$$
\begin{gathered}
\left.T_{t}\left(p_{s}\right)\right|_{P_{[i+2 q+1, j+2 q+1][i, j]}}=(-1)^{j} e_{i+2 q+1, j+2 q+1} \otimes e_{i, j} \quad(1 \leq j \leq n-3-2 q), \\
\left.T_{t}\left(p_{s}\right)\right|_{P_{[i+2 q+1, n-1][i, n-2-2 q]}}=-e_{i+2 q+1, n-1} \otimes \gamma_{i, n} \nu_{i, n-2-2 q} \\
-\sum_{z=\frac{n-2}{2}-q}^{\frac{n-2}{2}} \gamma_{i+2 q+1, n-1} \nu_{i+2 q+1,2 z+2 q-(n-3)} \otimes \alpha_{i, 2 z-1} \ldots \alpha_{i, n-2-2 q},
\end{gathered}
$$

$\left.T_{t}\left(p_{s}\right)\right|_{P_{[i+2 q+1, n][i, n-2-2 q]}}=-\sum_{z=\frac{n-2}{2}-q}^{\frac{n-4}{2}} \gamma_{i+2 q+1, n} \nu_{i+2 q+1,2 z+2 q-(n-4)} \otimes \alpha_{i, 2 z} \ldots \alpha_{i, n-2-2 q}$,

$\left.T_{t}\left(p_{s}\right)\right|_{P_{[i+2 q+2,2 j+2 q-(n-2)][i, 2 j]}}$

$$
\begin{aligned}
& =\sum_{z=j}^{\frac{n-2}{2}} \mu_{i+2 q+2,2 j+2 q-(n-1)} \tau_{i+2 q+1} \nu_{i+2 q+1,2 z+2 q-(n-3)} \otimes \alpha_{i, 2 z-1} \ldots \alpha_{i, 2 j} \\
& \quad-\sum_{z=\frac{n-2}{2}-q}^{j-1} \alpha_{i+2 q+2,2 j+2 q-(n-1)} \ldots \alpha_{i+2 q+2,2 z+2 q-(n-4)} \otimes \mu_{i+1,2 z} \tau_{i} \nu_{i, 2 j} \\
& \\
& \quad\left(\frac{n}{2}-q \leq j \leq \frac{n-2}{2}\right),
\end{aligned}
$$

$\left.T_{t}\left(p_{s}\right)\right|_{P_{[i+2 q+2,2 j+2 q-(n-3)][i, 2 j+1]}}$

$$
\begin{aligned}
= & -\sum_{z=j}^{\frac{n-4}{2}} \mu_{i+2 q+2,2 j+2 q-(n-2)} \tau_{i+2 q+1} \nu_{i+2 q+1,2 z+2 q-(n-4)} \otimes \alpha_{i, 2 z} \ldots \alpha_{i, 2 j+1} \\
& +\mu_{i+2 q+2,2 j+2 q-(n-2)} \beta_{i+2 q+1, n} \otimes \gamma_{i, n-1} \nu_{i, 2 j+1} \\
& +\sum_{z=\frac{n-2}{2}-q}^{j} \alpha_{i+2 q+2,2 j+2 q-(n-2)} \ldots \alpha_{i+2 q+2,2 z+2 q-(n-3)} \otimes \mu_{i+1,2 z-1} \tau_{i} \nu_{i, 2 j+1} \\
& \quad\left(\frac{n-2}{2}-q \leq j \leq \frac{n-4}{2}\right),
\end{aligned}
$$


$\left.T_{t}\left(p_{s}\right)\right|_{P_{[i+2 q+2,2 q+1][i, n]}}=0$,

$\left.T_{t}\left(p_{s}\right)\right|_{P_{[i+2 q+2,2 q+1][i, n-1]}}=\mu_{i+2 q+2,2 q} \beta_{i+2 q+1, n} \otimes e_{i, n-1}$

$$
+\sum_{z=\frac{n-2}{2}-q}^{\frac{n-2}{2}} \alpha_{i+2 q+2,2 q} \ldots \alpha_{i+2 q+2,2 z+2 q-(n-3)} \otimes \mu_{i+1,2 z-1} \beta_{i, n-1} ;
$$

4.4) if $t=4 q+1,0 \leq q \leq(n-4) / 2$, then

$$
\begin{aligned}
& \left.T_{t}\left(p_{s}\right)\right|_{P_{[i+2 q+1,2 j+2 q][i, 2 j]}} \\
& =-\sum_{z=j}^{\frac{n-4}{2}-q} \mu_{i+2 q+1,2 j+2 q-1} \tau_{i+2 q} \nu_{i+2 q, 2 z+2 q+1} \otimes \alpha_{i, 2 z-1} \ldots \alpha_{i, 2 j} \\
& \quad-\sum_{z=0}^{j-1} \alpha_{i+2 q+1,2 j+2 q-1} \ldots \alpha_{i+2 q+1,2 z+2 q+2} \otimes \mu_{i+1,2 z} \tau_{i} \nu_{i, 2 j} \\
& \quad\left(1 \leq j \leq \frac{n-2}{2}-q\right),
\end{aligned}
$$

$\left.T_{t}\left(p_{s}\right)\right|_{P_{[i+2 q+1,2 j+2 q+1][i, 2 j+1]}}$

$$
\begin{aligned}
& =\sum_{z=j}^{\frac{n-4}{2}-q} \mu_{i+2 q+1,2 j+2 q} \tau_{i+2 q} \nu_{i+2 q, 2 z+2 q+2} \otimes \alpha_{i, 2 z} \ldots \alpha_{i, 2 j+1} \\
& \quad+\alpha_{i+2 q+1,2 j+2 q} \ldots \alpha_{i+2 q+1,2 q+1} \otimes \gamma_{i, n-1} \nu_{i, 2 j+1}
\end{aligned}
$$

$\left.T_{t}\left(p_{s}\right)\right|_{P_{[i+2 q+1, n-1][i, n-1]}}=-\gamma_{i+2 q+1, n-1} \nu_{i+2 q+1,2 q+1} \otimes e_{i, n-1}$

$$
\begin{aligned}
& -\sum_{z=1}^{\frac{n-4}{2}-q} \gamma_{i+2 q+1, n-1} \nu_{i+2 q+1,2 z+2 q+1} \otimes \mu_{i+1,2 z-1} \beta_{i, n-1} \\
& -e_{i+2 q+1, n-1} \otimes \mu_{i+1, n-3-2 q} \beta_{i, n-1},
\end{aligned}
$$

$\left.T_{t}\left(p_{s}\right)\right|_{P_{[i+2 q+1, n][i, n]}}=\sum_{z=0}^{\frac{n-4}{2}-q} \gamma_{i+2 q+1, n} \nu_{i+2 q+1,2 z+2 q+2} \otimes \mu_{i+1,2 z} \beta_{i, n}$,

$\left.T_{t}\left(p_{s}\right)\right|_{P_{[i+2 q+1, j+2 q-(n-2)][i, j]}}=(-1)^{j} e_{i+2 q+1, j+2 q-(n-2)} \otimes e_{i, j}$

$$
(n-1-2 q \leq j \leq n-2)
$$

4.5) if $t=4 q+3,0 \leq q \leq(n-4) / 2$, then

$$
\begin{gathered}
\left.T_{t}\left(p_{s}\right)\right|_{P_{[i+2 q+2,2 j+2 q+1][i, 2 j]}} \\
=\sum_{z=j}^{\frac{n-4}{2}-q} \begin{array}{r}
\mu_{i+2 q+2,2 j+2 q} \tau_{i+2 q+1} \nu_{i+2 q+1,2 z+2 q+2} \otimes \alpha_{i, 2 z-1} \ldots \alpha_{i, 2 j} \\
+\mu_{i+2 q+2,2 j+2 q} \beta_{i+2 q+1, n} \otimes \alpha_{i, n-4-2 q} \ldots \alpha_{i, 2 j} \\
-\mu_{i+2 q+2,2 j+2 q} \beta_{i+2 q+1, n-1} \otimes \alpha_{i, n-4-2 q} \ldots \alpha_{i, 2 j}
\end{array} \\
\quad+\sum_{z=0}^{j-1} \alpha_{i+2 q+2,2 j+2 q} \ldots \alpha_{i+2 q+2,2 z+2 q+3} \otimes \mu_{i+1,2 z} \tau_{i} \nu_{i, 2 j}
\end{gathered}
$$$$
\left(1 \leq j \leq \frac{n-4}{2}-q\right),
$$ 


$$
\begin{aligned}
& \left.T_{t}\left(p_{s}\right)\right|_{P_{[i+2 q+2,2 j+2 q+2][i, 2 j+1]}} \\
& =-\sum_{z=j}^{\frac{n-6}{2}-q} \begin{array}{r}
\mu_{i+2 q+2,2 j+2 q+1} \tau_{i+2 q+1} \nu_{i+2 q+1,2 z+2 q+3} \otimes \alpha_{i, 2 z} \ldots \alpha_{i, 2 j+1} \\
-\mu_{i+2 q+2,2 j+2 q+1} \beta_{i+2 q+1, n-1} \otimes \alpha_{i, n-4-2 q} \ldots \alpha_{i, 2 j+1}
\end{array} \\
& \begin{array}{c}
-\alpha_{i+2 q+2,2 j+2 q+1} \ldots \alpha_{i+2 q+2,2 q+2} \otimes \gamma_{i, n-1} \nu_{i, 2 j+1} \\
-\sum_{z=1}^{j} \alpha_{i+2 q+2,2 j+2 q+1} \ldots \alpha_{i+2 q+2,2 z+2 q+2} \otimes \mu_{i+1,2 z-1} \tau_{i} \nu_{i, 2 j+1}
\end{array} \\
& \left(0 \leq j \leq \frac{n-4}{2}-q\right), \\
& \left.T_{t}\left(p_{s}\right)\right|_{P_{[i+2 q+2, n][i, n-1]}}=\gamma_{i+2 q+2, n} \nu_{i+2 q+2,2 q+2} \otimes e_{i, n-1} \\
& +\sum_{z=1}^{\frac{n-4}{2}-q} \gamma_{i+2 q+2, n} \nu_{i+2 q+2,2 z+2 q+2} \otimes \mu_{i+1,2 z-1} \beta_{i, n-1}, \\
& \left.T_{t}\left(p_{s}\right)\right|_{P_{[i+2 q+2, n-1][i, n]}}=-\sum_{z=0}^{\frac{n-6}{2}-q} \gamma_{i+2 q+2, n-1} \nu_{i+2 q+2,2 z+2 q+3} \otimes \mu_{i+1,2 z} \beta_{i, n} \\
& \left.T_{t}\left(p_{s}\right)\right|_{P_{[i+2 q+2, j+2 q-(n-3)][i, j]}}=(-1)^{j+1} e_{i+2 q+2, j+2 q-(n-3)} \otimes e_{i, j} \\
& (n-2-2 q \leq j \leq n-2) \text {; }
\end{aligned}
$$

4.6) if $t=u+v(2 n-3)$ with $0 \leq u \leq 2 n-4$ and $v \geq 1$, then $T_{t}\left(p_{s}\right)=\sigma^{v}\left(T_{u}\left(p_{s}\right)\right)$, where $T_{u}\left(p_{s}\right)$ is described by the formulas in 4.1)-4.5).

Proof. We need to verify that, for $b \in\left\{f_{s}, g_{s}, h_{s}, p_{s}\right\}$, the identities $\mu T_{0}(b)=b$ and $d_{t} T_{t+1}(b)=T_{t}(b) d_{t+s}$ are valid for all $t \geq 0$. If $t=u+v(2 n-3)$, where $0 \leq u \leq 2 n-4$ and $v \geq 1$, then $d_{t} T_{t+1}(b)=\sigma^{v}\left(d_{u} T_{u+1}(b)\right), T_{t}(b) d_{t+s}=\sigma^{v}\left(T_{u}(b) d_{u+s}\right)$. Hence, we need to prove that $d_{t} T_{t+1}(b)=T_{t}(b) d_{t+s}$ only for all $0 \leq t \leq 2 n-4$. For this, we consider the cases 1.1)-1.4), 2.1)-2.5), 3.1)-3.5), 4.1)-4.5) separately. Then, by using the formulas for the differentials $d_{t}$ given in 9], the required identities are proved by direct but cumbersome calculations. We leave this to the reader.

Remark 3. It is clear that $f_{0}$ is the unity of the algebra $\mathrm{HH}^{*}(R)$. Moreover, the resolution described in Theorem 1 is $\lambda$-periodic with $\lambda$ as in (2.1), and hence, $T_{t}\left(f_{\lambda}\right)$ is an isomorphism for all $t \geq 0$. Consequently, multiplication by $f_{\lambda}$ gives rise to an isomorphism between $\mathrm{HH}^{s}(R)$ and $\mathrm{HH}^{s+\lambda}(R)$ for all $s \geq 1$.

\section{$\S 4$. Generators}

In this section, we describe a set of generators for the algebra $\operatorname{HH}^{*}(R)$. This set is infinite, but later we show how to pick a finite subset in it generating $\mathrm{HH}^{*}(R)$.

First, we define some additional elements of $\mathrm{HH}^{*}(R)$.

We define a 1-cocycle $\varepsilon_{1} \in \operatorname{Ker} \delta^{1}$ as follows:

$$
\varepsilon_{1}=u_{1,0, n-1}+u_{1,0, n} .
$$

Assume that $s=l(2 n-3), l \geq 1, r|l(n-1)-1,2| l n$, and either char $k=2$ or $2 \nmid l$. Let $\chi_{s}$ (respectively $\chi_{s}^{\prime}$ ) be the $s$-cocycle equal to $v_{r, n}$ on $P_{[1, n][r, n]}$ (respectively, to $v_{r, n-1}$ on $\left.P_{[1, n-1][r, n-1]}\right)$ and to 0 on the remaining direct summands of $Q_{s}$.

Assume that $s=l(2 n-3), r \mid l(n-1)-1$ and $2 \nmid l n$. Let $\xi_{s}$ be the $s$-cocycle equal to $t_{r, n-2,1}$ on $P_{[1,1][r, 1]}$ and to 0 on the remaining summands of $Q_{s}$.

If $r=1$, then, for simplicity, we shall omit the first index in notation such as $e_{i, j}$ and $P_{i, j}$. If $1 \leq j \leq n-2$, then we denote by $\varepsilon_{0}^{(j)}$ the 0 -cocycle equal to $t_{n-2, j}$ on $P_{[j][j]}$ and 
to 0 on the remaining direct summands of $Q_{0}$. If $q \in\{n-1, n\}$, then we denote by $\varepsilon_{0}^{(q)}$ the 0-cocycle equal to $v_{q}$ on $P_{[q][q]}$ and to 0 on the remaining direct summands of $Q_{0}$.

The proofs of Theorems 2 and 3 in [9] show that the maps $\varepsilon_{1}, \chi_{s}, \chi_{s}^{\prime}, \xi_{s}$, and $\varepsilon_{0}^{(j)}$ defined above are indeed cocycles.

Remark 4. Let $s=l(2 n-3)$ with $l \geq 1$. It is clear that the elements $\chi_{s}$ and $\chi_{s}^{\prime}$ are defined if and only if the element $f_{s-1}$ is defined, and the element $\xi_{s}$ is defined if and only if the element $h_{s-1}$ is defined. Moreover, if $\chi_{s}$ and $\chi_{s}^{\prime}$ are defined, then $p_{s-1}$ is defined if and only if $2 \mid n$.

Lemma 1. 1) Assume that $s$ is a number such that the element $f_{s}$ is defined and $2 n-3 \chi$ $s+1$. Then $\varepsilon_{1} f_{s} \neq 0$ in $\mathrm{HH}^{*}(R)$.

2) Assume that $s$ is such that $g_{s}$ is defined. Then $\varepsilon_{1} g_{s} \neq 0$ in $\mathrm{HH}^{*}(R)$.

3) Assume that $s$ is such that $h_{s}$ is defined. Then in $\mathrm{HH}^{*}(R)$ we have

$$
\varepsilon_{1} h_{s}=\left(\frac{n-1}{2}\right) \xi_{s+1} .
$$

Moreover, $\xi_{s+1} \neq 0$ in $\mathrm{HH}^{*}(R)$.

4) Assume that $s$ is such that $f_{s}$ and $p_{s}$ are defined. Then in $\mathrm{HH}^{*}(R)$ we have

$$
\begin{aligned}
\varepsilon_{1} f_{s} & =\chi_{s+1}-\chi_{s+1}^{\prime}, \\
\varepsilon_{1} p_{s} & =\left(\frac{2-n}{2}\right) \chi_{s+1}-\left(\frac{n}{2}\right) \chi_{s+1}^{\prime} .
\end{aligned}
$$

Moreover, $\chi_{s+1}$ and $\chi_{s+1}^{\prime}$ are linearly independent over $k$ in $\operatorname{HH}^{*}(R)$.

5) Assume that $s$ is such that the element $\chi_{s}$ is defined and $2 \nmid n$. Then $\chi_{s} \neq 0$ in $\mathrm{HH}^{*}(R)$.

Proof. 1) As was noticed at the beginning of the previous section, the element $\varepsilon_{1} f_{s} \in$ $\mathrm{HH}^{*}(R)$ is determined by the cocycle $\mu T_{0}\left(\varepsilon_{1}\right) T_{1}\left(f_{s}\right)=\varepsilon_{1} T_{1}\left(f_{s}\right)$. Using the formulas of item 1.2) of Proposition 11 we see that $\varepsilon_{1} T_{1}\left(f_{s}\right)=u_{1, m, n-1}+u_{1, m, n}$. We must prove that $\varepsilon_{1} T_{1}\left(f_{s}\right) \notin \operatorname{Im} \delta^{s}$. Assume that this is not true. Then there exists a homomorphism $w \in \operatorname{Hom}_{\Lambda}\left(Q_{s}, R\right)$ such that $\delta^{s}(w)=u_{1, m, n-1}+u_{1, m, n}$. The proofs of Theorems 2 and 3] in [9] show that $w=\sum_{i=1}^{r}\left(\sum_{j=1}^{n-2-m} \varkappa_{i, j} w_{i, m, j}+\varphi_{i, 1} u_{i, n-1}+\varphi_{i, 2} u_{i, n}\right)+w^{\prime}$ for some $\varkappa_{i, j}, \varphi_{i, 1}, \varphi_{i, 2} \in k$, where $w^{\prime}=0$ for $r>1$ and $w^{\prime} \in \operatorname{Ker} \delta^{s}$ for $r=1$. From the same proofs, it follows that if $\delta^{s}(w)=u_{1, m, n-1}+u_{1, m, n}$, then

$$
\begin{aligned}
& \varkappa_{i, j+1}-\varkappa_{i, j}=0, \quad \varkappa_{i+1,1}-\varphi_{i, 1}=0, \quad \varkappa_{i+1,1}-\varphi_{i, 2}=0 \quad(1 \leq j \leq n-3-m, 1 \leq i \leq r) ; \\
& \varphi_{i, 1}-\varkappa_{i, n-2-m}=0, \quad \varphi_{i, 2}-\varkappa_{i, n-2-m}=0 \quad(2 \leq i \leq r) ; \\
& \varphi_{1,1}-\varkappa_{1, n-2-m}=1, \quad \varphi_{1,2}-\varkappa_{1, n-2-m}=1 .
\end{aligned}
$$

The first two lines imply that $\varphi_{i+1,1}=\varphi_{i, 1}$ for $1 \leq i \leq r-1$, i.e., we have $\varphi_{r, 1}=\varphi_{1,1}$. But the first and third lines show that, similarly, $\varphi_{1,1}=\varphi_{r, 1}+1$. Hence, we arrive at a contradiction, and this completes the proof of part 1 .

2) Using formulas of item 2.2) of Proposition 1, we obtain $\varepsilon_{1} T_{1}\left(g_{s}\right)=v_{r, n-1}$. Assume that $\varepsilon_{1} g_{s}=0$ in $\operatorname{HH}^{*}(R)$, i.e., there exists a homomorphism $w \in \operatorname{Hom}_{\Lambda}\left(Q_{s}, R\right)$ such that $\delta^{s}(w)=v_{r, n-1}$.

The proofs of Theorems 2 and 3 in [9] show that

$$
\begin{aligned}
w=\sum_{i=1}^{r}\left(\sum_{j=1}^{n-3-m} \varkappa_{i, j} w_{i, m+1, j}+\varphi_{i, 1}\right. & u_{i, m, n}+\varphi_{i, 2} u_{i, m, n-1} \\
& \left.+\sum_{j=n-1-m}^{n-2} \eta_{i, j} t_{i, m, j}+\zeta_{i, 1} v_{i, m, n-1}+\zeta_{i, 2} v_{i, m, n}\right)
\end{aligned}
$$


for some $\varkappa_{i, j}, \varphi_{i, q}, \eta_{i, j}, \zeta_{i, q} \in k$. The same proofs imply that the identity $\delta^{s}(w)=v_{r, n-1}$ is equivalent to the fact that

$$
\begin{aligned}
& \zeta_{i, 1}-\zeta_{i, 2}=\eta_{i, n-2}=\cdots=\eta_{i, n-1-m}=\varphi_{i, 2}-\varphi_{i, 1}, \\
& -\zeta_{i, 2}-\sum_{j=1}^{n-3-m} \varkappa_{i+1, j}+\varphi_{i+1,1}=0 \quad(1 \leq i \leq r) ; \\
& -\zeta_{i, 1}-\sum_{j=1}^{n-3-m} \varkappa_{i+1, j}+\varphi_{i+1,2}=0 \quad(1 \leq i \leq r-1) ; \\
& -\zeta_{r, 1}-\sum_{j=1}^{n-3-m} \varkappa_{1, j}+\varphi_{1,2}=1 .
\end{aligned}
$$

Put $\epsilon_{i}=\zeta_{i, 1}-\zeta_{i, 2}=\varphi_{i, 2}-\varphi_{i, 1}$. Then, by the second and third line, we have $\epsilon_{i+1}=\epsilon_{i}$ for $1 \leq i \leq r-1$, i.e., $\epsilon_{r}=\epsilon_{1}$. But the second and fourth lines imply that $\epsilon_{1}=\epsilon_{r}+1$. This contradiction completes the proof of part 2).

3) We obtain $\varepsilon_{1} T_{1}\left(h_{s}\right)=\sum_{z=0}^{\frac{n-3}{2}} t_{1, n-2,2 z+1}$ with the help of formulas of item 3.4) of Proposition 1. By definition, we have $\xi_{s+1}=t_{r, n-2,1}$. By the proofs of Theorems 2 and 3 in [9], the set $\left\{t_{i, n-2, j}\right\}_{1 \leq i \leq r, 1 \leq j \leq n-2}$ is a $k$-basis of $\operatorname{Ker} \delta^{s+1}$.

Now we find a condition on the elements $\eta_{i, j} \in k$ under which the element $v=$ $\sum_{i=1}^{r} \sum_{j=1}^{n-2} \eta_{i, j} t_{i, n-2, j} \in \operatorname{Ker} \delta^{s+1}$ equals 0 in $\operatorname{HH}^{*}(R)$, i.e., there exists $w \in \operatorname{Hom}_{\Lambda}\left(Q_{s}, R\right)$ such that $\delta^{s}(w)=v$. Assume that such $w$ exists. From the proofs of Theorems 2and 3 in [9], it is clear that $w=\sum_{i=1}^{r} \sum_{j=1}^{n-2} \varkappa_{i, j} w_{i, 0, j}+w^{\prime}$ for some $\varkappa_{i, j} \in k$, where $w^{\prime}=0$ for $r>1$ and $w^{\prime} \in \operatorname{Ker} \delta^{s}$ for $r=1$. The same proofs imply that $\sum_{z=j}^{n-2} \varkappa_{i, z}-\sum_{z=1}^{j} \varkappa_{i+1, z}=\eta_{i, j}$ with $1 \leq i \leq r$ and $1 \leq j \leq n-2$. Adding all these identities, we get $\sum_{i=1}^{r} \sum_{j=1}^{n-2} \eta_{i, j}=0$.

This condition defines a subspace $V \subset \operatorname{Ker} \delta^{s+1}$ of dimension $r(n-2)-1$. Since $\operatorname{dim}_{k} \operatorname{Im} \delta^{s}=r(n-2)-1$ and $\operatorname{Im} \delta^{s} \subset V$, we obtain

$$
\operatorname{Im} \delta^{s}=V=\left\{\sum_{i=1}^{r} \sum_{j=1}^{n-2} \eta_{i, j} t_{i, n-2, j} \mid \sum_{i=1}^{r} \sum_{j=1}^{n-2} \eta_{i, j}=0\right\} .
$$

Clearly, $\sum_{z=0}^{\frac{n-3}{2}} t_{1, n-2,2 z+1}-\left(\frac{n-1}{2}\right) t_{r, n-2,1} \in \operatorname{Im} \delta^{s}$ and $t_{r, n-2,1} \notin \operatorname{Im} \delta^{s}$, i.e., we have $\varepsilon_{1} h_{s}=\left(\frac{n-1}{2}\right) \xi_{s+1}$ and $\xi_{s+1} \neq 0$ in $\operatorname{HH}^{*}(R)$.

4) Using formulas of items 1.3) and 4.4) of Proposition 11 we see that $\varepsilon_{1} T_{1}\left(f_{s}\right)=$ $v_{r, n}-v_{r, n-1}$ and $\varepsilon_{1} T_{1}\left(p_{s}\right)=-\sum_{z=1}^{\frac{n-2}{2}} t_{1, n-2,2 z}-v_{r, n-1}$. By definition, we have $\chi_{s+1}=v_{r, n}$ and $\chi_{s+1}^{\prime}=v_{r, n-1}$. Hence, $\varepsilon_{1} T_{1}\left(f_{s}\right)=\chi_{s+1}-\chi_{s+1}^{\prime}$, i.e., $\varepsilon_{1} f_{s}=\chi_{s+1}-\chi_{s+1}^{\prime}$ in $\operatorname{HH}^{*}(R)$. The proofs of Theorems 2 and 3 in $[9$ show that the set

$$
\left\{t_{i, n-2, j}\right\}_{1 \leq i \leq r, 1 \leq j \leq n-2} \cup\left\{v_{i, q}\right\}_{1 \leq i \leq r, q \in\{n-1, n\}}
$$

is a $k$-basis of $\operatorname{Ker} \delta^{s+1}$ for $r>1$. If $r=1$, the set

$$
\left\{t_{1, n-2, j}\right\}_{1 \leq j \leq n-2} \cup\left\{v_{1, q}\right\}_{q \in\{n-1, n\}} \cup\left\{w_{0}\right\}
$$

with a suitable $w_{0}$ in $\operatorname{Ker} \delta^{s+1}$ forms a $k$-basis of $\operatorname{Ker} \delta^{s+1}$.

Now we find a condition on the elements $\eta_{i, j}, \zeta_{i, n-1}, \zeta_{i, n}, \epsilon \in k$ under which the element

$$
v=\sum_{i=1}^{r}\left(\sum_{j=1}^{n-2} \eta_{i, j} t_{i, n-2, j}+\zeta_{i, n-1} v_{i, n-1}+\zeta_{i, n} v_{i, n}\right)+\epsilon w_{0} \in \operatorname{Ker} \delta^{s+1}
$$

is equal to 0 in $\operatorname{HH}^{*}(R)$, i.e., there exists $w \in \operatorname{Hom}_{\Lambda}\left(Q_{s}, R\right)$ such that $\delta^{s}(w)=v$ (for $r>1$ we put $w_{0}=0$ and $\left.\epsilon=0\right)$. Assume that such $w$ exists. The proofs of Theorems 2 
and 3 in 9 show that

$$
w=\sum_{i=1}^{r}\left(\sum_{j=1}^{n-2} \varkappa_{i, j} w_{i, 0, j}+\varphi_{i, n-1} u_{i, n-1}+\varphi_{i, n} u_{i, n}\right)+w^{\prime}
$$

with $\varkappa_{i, j}, \varphi_{i, n-1}, \varphi_{i, n} \in k$, where $w^{\prime}=0$ for $r>1$ and $w^{\prime} \in \operatorname{Ker} \delta^{s}$ for $r=1$. The same proofs imply the following identities:

$$
\begin{aligned}
\sum_{z=j}^{n-2} \varkappa_{i, z}-\varphi_{i, n-1}+\varphi_{i, n}-\sum_{z=1}^{j} \varkappa_{i+1, z} & =\eta_{i, j} & & (1 \leq i \leq r, 1 \leq j \leq n-2) ; \\
\varphi_{i, n-1}+\sum_{z=1}^{n-2} \varkappa_{i+1, z}-\varphi_{i+1, n-1} & =\zeta_{i, n-1} & & (1 \leq i \leq r) ; \\
-\varphi_{i, n}+\sum_{z=1}^{n-2} \varkappa_{i+1, z}+\varphi_{i+1, n} & =\zeta_{i, n} & & (1 \leq i \leq r) .
\end{aligned}
$$

If $r=1$, then we also have $\epsilon=0$. We subtract the third identity from the second. Then, summing the identities obtained for $1 \leq i \leq r$, we get $\sum_{i=1}^{r}\left(\zeta_{i, n-1}-\zeta_{i, n}\right)=0$. The first and second identities imply $\sum_{i=1}^{r}\left(\sum_{j=1}^{n-2}(-1)^{j} \eta_{i, j}+\zeta_{i, n-1}\right)=0$. Clearly, the system

$$
\left\{\begin{array}{l}
\epsilon=0, \\
\sum_{i=1}^{r}\left(\zeta_{i, n-1}-\zeta_{i, n}\right)=0, \\
\sum_{i=1}^{r}\left(\sum_{j=1}^{n-2}(-1)^{j} \eta_{i, j}+\zeta_{i, n-1}\right)=0
\end{array}\right.
$$

determines a subspace $V \subset \operatorname{Ker} \delta^{s+1}$ of dimension $r n-2$. Since $\operatorname{dim}_{k} \operatorname{Im} \delta^{s}=r n-2$ and $\operatorname{Im} \delta^{s} \subset V$, we have

$$
\begin{aligned}
\operatorname{Im} \delta^{s}=V=\left\{\sum_{i=1}^{r}\left(\sum_{j=1}^{n-2} \eta_{i, j} t_{i, n-2, j}+\zeta_{i, n-1} v_{i, n-1}+\zeta_{i, n} v_{i, n}\right)\right. \\
\left.\sum_{i=1}^{r}\left(\zeta_{i, n-1}-\zeta_{i, n}\right)=0, \sum_{i=1}^{r}\left(\sum_{j=1}^{n-2}(-1)^{j} \eta_{i, j}+\zeta_{i, n-1}\right)=0\right\} .
\end{aligned}
$$

It is easily seen that if $y_{1} v_{r, n}+y_{2} v_{r, n-1} \in \operatorname{Im} \delta^{s}$ with $y_{1}, y_{2} \in k$, then $y_{1}=y_{2}=0$, i.e., the elements $\chi_{s+1}$ and $\chi_{s+1}^{\prime}$ are linearly independent over $k$ in $\operatorname{HH}^{*}(R)$.

Moreover, it is easy to verify that $-\sum_{z=1}^{\frac{n-2}{2}} t_{1, n-2,2 z}-v_{r, n-1}+\left(\frac{n-2}{2}\right) v_{r, n}+\left(\frac{n}{2}\right) v_{r, n-1} \in$ $\operatorname{Im} \delta^{s}$; hence, in $\operatorname{HH}^{*}(R)$ we have $\varepsilon_{1} p_{s}=\left(\frac{2-n}{2}\right) \chi_{s+1}-\left(\frac{n}{2}\right) \chi_{s+1}^{\prime}$.

5) Observe that $\chi_{s}=v_{r, n}$. The proofs of Theorems 2 and 3 in 9 show that the set $\left\{t_{i, n-2, j}\right\}_{1 \leq i \leq r, 1 \leq j \leq n-2} \cup\left\{v_{i, q}\right\}_{1 \leq i \leq r, q \in\{n-1, n\}}$ is a $k$-basis of $\operatorname{Ker} \delta^{s}$ for $r>1$, and for $r=1$, the set $\left\{t_{1, n-2, j}\right\}_{1 \leq j \leq n-2} \cup\left\{v_{1, q}\right\}_{q \in\{n-1, n\}} \cup\left\{w_{0}\right\}$, where $w_{0}$ is a suitable element of $\operatorname{Ker} \delta^{s}$, is a $k$-basis of $\operatorname{Ker} \delta^{s}$.

Now we derive a condition on the elements $\eta_{i, j}, \zeta_{i, n-1}, \zeta_{i, n}, \epsilon \in k$ equivalent to the fact that the element

$$
v=\sum_{i=1}^{r}\left(\sum_{j=1}^{n-2} \eta_{i, j} t_{i, n-2, j}+\zeta_{i, n-1} v_{i, n-1}+\zeta_{i, n} v_{i, n}\right)+\epsilon w_{0} \in \operatorname{Ker} \delta^{s}
$$

is equal to 0 in $\operatorname{HH}^{*}(R)$, i.e., there exists $w \in \operatorname{Hom}_{\Lambda}\left(Q_{s-1}, R\right)$ such that $\delta^{s-1}(w)=v$ (if $r>1$, we put $w_{0}=0$ and $\left.\epsilon=0\right)$. Assume that such $w$ exists. By the proofs of Theorems 2 and 3 in 9 , we can write

$$
w=\sum_{i=1}^{r}\left(\sum_{j=1}^{n-2} \varkappa_{i, j} w_{i, 0, j}+\varphi_{i, n-1} u_{i, n-1}+\varphi_{i, n} u_{i, n}\right)+w^{\prime}
$$


with $\varkappa_{i, j}, \varphi_{i, n-1}, \varphi_{i, n} \in k$, where $w^{\prime}=0$ for $r>1$ and $w^{\prime} \in \operatorname{Ker} \delta^{s-1}$ for $r=1$. Also, the same proofs imply the identities

$$
\begin{aligned}
\sum_{z=j}^{n-2} \varkappa_{i, z}-\varphi_{i, n-1}+\varphi_{i, n}-\sum_{z=1}^{j} \varkappa_{i+1, z} & =\eta_{i, j} & & (1 \leq i \leq r, 1 \leq j \leq n-2) ; \\
\varphi_{i, n-1}+\sum_{z=1}^{n-2} \varkappa_{i+1, z}-\varphi_{i+1, n-1} & =\zeta_{i, n-1} & & (1 \leq i \leq r) ; \\
-\varphi_{i, n}+\sum_{z=1}^{n-2} \varkappa_{i+1, z}+\varphi_{i+1, n} & =\zeta_{i, n} & & (1 \leq i \leq r) .
\end{aligned}
$$

If $r=1$, then $\epsilon=0$. We add the third identity to the second. Then, summing the identities obtained for $1 \leq i \leq r$, we see that $\sum_{i=1}^{r}\left(\zeta_{i, n-1}+\zeta_{i, n}\right)=0$. Clearly, the system

$$
\left\{\begin{array}{l}
\epsilon=0 \\
\sum_{i=1}^{r}\left(\zeta_{i, n-1}+\zeta_{i, n}\right)=0
\end{array}\right.
$$

determines a subspace $V \subset \operatorname{Ker} \delta^{s}$ of dimension $r n-1$. Since $\operatorname{dim}_{k} \operatorname{Im} \delta^{s-1}=r n-1$ and $\operatorname{Im} \delta^{s-1} \subset V$, we have

$$
\begin{aligned}
\operatorname{Im} & \delta^{s-1}=V \\
& =\left\{\sum_{i=1}^{r}\left(\sum_{j=1}^{n-2} \eta_{i, j} t_{i, n-2, j}+\zeta_{i, n-1} v_{i, n-1}+\zeta_{i, n} v_{i, n}\right) \mid \sum_{i=1}^{r}\left(\zeta_{i, n-1}+\zeta_{i, n}\right)=0\right\} .
\end{aligned}
$$

It follows that $\chi_{s} \neq 0$ in $\mathrm{HH}^{*}(R)$. The lemma is proved.

Remark 5. In the sequel, we use the following easily proved assertion: if $v \in \operatorname{Ker} \delta^{s}$ and $v\left(Q_{s}\right) \not \subset \operatorname{Rad} R$, then $v \neq 0$ in $\operatorname{HH}^{*}(R)$.

Corollary 1. If the element $f_{s}$ (respectively, $g_{s}$ or $\left.h_{s}\right)$ is defined for some $s \geq 0$, then $f_{s}$ (respectively, $g_{s}$ or $\left.h_{s}\right)$ is nonzero in $\mathrm{HH}^{*}(R)$. Moreover, if $p_{s}$ and $f_{s}$ are defined for a fixed $s$, then they are linearly independent over $k$ in $\mathrm{HH}^{*}(R)$.

Proof. The fact that $g_{s} \neq 0$ follows easily from Lemma 1. All remaining assertions follow from Remark 5 .

We consider the following sets (in each case, we include a variable with index $s$ in the corresponding set if the variable is defined for this $s$ ):

$\mathcal{F}=\left\{\varepsilon_{1}, f_{s}, g_{s}\right\} ; \quad \mathcal{G}_{1}=\left\{p_{s}\right\} ; \quad \mathcal{G}_{2}=\left\{h_{s}\right\} ; \quad \mathcal{H}_{1}=\left\{\chi_{s}\right\} ; \mathcal{H}_{2}=\left\{\xi_{s}\right\} ; \quad \mathcal{J}=\left\{\varepsilon_{0}^{(j)}\right\}_{1 \leq j \leq n}$.

Next, we put

$$
\begin{array}{lll}
\mathcal{Z}_{1}=\mathcal{F} \cup \mathcal{G}_{1} ; & \mathcal{Z}_{2}=\mathcal{Z}_{1} \cup \mathcal{H}_{1} ; & \mathcal{Z}_{3}=\mathcal{F} \cup \mathcal{G}_{2} ; \\
\mathcal{Z}_{4}=\mathcal{Z}_{3} \cup \mathcal{H}_{2} ; & \mathcal{Z}_{5}=\mathcal{Z}_{3} \cup \mathcal{H}_{1} ; & \mathcal{Z}_{6}=\mathcal{Z}_{3} \cup \mathcal{H}_{1} \cup \mathcal{H}_{2} .
\end{array}
$$

Proposition 2. 1) Assume that $2 \mid n$, char $k \nmid n-1$, and $r>1$. Then the set $\mathcal{Z}_{1}$ generates $\mathrm{HH}^{*}(R)$ as a k-algebra.

2) Assume that $2 \mid n$, char $k \mid n-1$, and $r>1$. Then the set $\mathcal{Z}_{2}$ generates $\operatorname{HH}^{*}(R)$ as a k-algebra.

3) Assume that $2 \nmid n$, char $k \neq 2$, char $K \nmid\left(\frac{n-1}{2}\right)$, and $r>1$. Then the set $\mathcal{Z}_{3}$ generates $\mathrm{HH}^{*}(R)$ as a $k$-algebra.

4) Assume that $2 \nmid n$, char $k \neq 2$, char $k \mid\left(\frac{n-1}{2}\right)$, and $r>1$. Then the set $\mathcal{Z}_{4}$ generates $\mathrm{HH}^{*}(R)$ as a $k$-algebra. 
5) Assume that $2 \nmid n$, char $k=2$, char $k \nmid\left(\frac{n-1}{2}\right)$, and $r>1$. Then the set $\mathcal{Z}_{5}$ generates $\mathrm{HH}^{*}(R)$ as a k-algebra.

6) Assume that $2 \nmid n$, char $k=2$, char $k \mid\left(\frac{n-1}{2}\right)$, and $r>1$. Then the set $\mathcal{Z}_{6}$ generates $\mathrm{HH}^{*}(R)$ as a $k$-algebra.

7) If we replace the condition $r>1$ by the condition $r=1$ in any of items 1$)-6)$, then the set $\mathcal{Z}_{i} \cup \mathcal{J}$, where $i$ is the number of the corresponding item, generates $\operatorname{HH}^{*}(R)$ as a $k$-algebra.

Proof. Let $\mathcal{H}$ denote a subalgebra of $\operatorname{HH}^{*}(R)$ generated by the corresponding set $\mathcal{Z}_{i}$ for a fixed item among 1)-6) and, respectively, generated by the set $\mathcal{Z}_{i} \cup \mathcal{J}$ for part 7). Fix any $s \geq 0$. We prove that $\operatorname{HH}^{s}(R) \subset \mathcal{H}$.

a) First we consider the case where $r>1$. We may assume that $s$ satisfies one of conditions (2.2) -(2.6). We analyze all cases successively.

1. Assume that $s=2 m+l(2 n-3)$ satisfies condition (2.2). Then the element $f_{s}$ is defined. We have $f_{s} \neq 0$ by Corollary 1, and $\operatorname{dim}_{k} \mathrm{HH}^{s}(R)=1$; hence, $f_{s}$ generates $\mathrm{HH}^{s}(R)$.

2. Assume that $s=2 m+1+l(2 n-3)$ satisfies condition (2.2). Then the element $f_{s-1}$ is defined. We have $\varepsilon_{1} f_{s-1} \neq 0$ by Lemma 1, and $\operatorname{dim}_{k} \operatorname{HH}^{s}(R)=1$; hence, $\varepsilon_{1} f_{s-1}$ generates $\mathrm{HH}^{s}(R)$.

3. Assume that $s=2 m+1+l(2 n-3)$ satisfies condition (2.3). Then the element $g_{s}$ is defined. We have $g_{s} \neq 0$ by Corollary 1, and $\operatorname{dim}_{k} \mathrm{HH}^{s}(R)=1$; hence, $g_{s}$ generates $\mathrm{HH}^{s}(R)$.

4. Assume that $s=2 m+2+l(2 n-3)$ satisfies condition (2.3). Then the element $g_{s-1}$ is defined. We have $\varepsilon_{1} g_{s-1} \neq 0$ by Lemma 11 and $\operatorname{dim}_{k} \operatorname{HH}^{s}(R)=1$; hence, $\varepsilon_{1} g_{s-1}$ generates $\mathrm{HH}^{s}(R)$.

5. Assume that $s=(l+1)(2 n-3)-1$ satisfies condition (2.4). Then the element $f_{s}$ is defined. Since $f_{s} \neq 0$ by Corollary 1 and $\operatorname{dim}_{k} \mathrm{HH}^{s}(R)=1$, we see that $f_{s}$ generates $\mathrm{HH}^{s}(R)$.

6. Assume that $s=l(2 n-3)$ satisfies condition (2.4). Then the element $\chi_{s}$ is defined and the assumption of one of items 5) and 6) is satisfied. Since $\chi_{s} \neq 0$ by Lemma 1 and $\operatorname{dim}_{k} \mathrm{HH}^{s}(R)=1$, we see that $\chi_{s}$ generates $\mathrm{HH}^{s}(R)$.

7. Assume that $s=(l+1)(2 n-3)-1$ satisfies condition (2.5). Then the element $h_{s}$ is defined and the assumption of one of items 3$)-6)$ is satisfied. Since $h_{s} \neq 0$ by Corollary 1 and $\operatorname{dim}_{k} \mathrm{HH}^{s}(R)=1$, we see that $h_{s}$ generates $\mathrm{HH}^{s}(R)$.

8. Assume that $s=l(2 n-3)$ satisfies condition (2.5). Then the elements $h_{s-1}$ and $\xi_{s}$ are defined and the assumption of one of items 3)-6) is satisfied. We have $\operatorname{dim}_{k} \mathrm{HH}^{s}(R)=$ 1 in this case. If the assumption of one of items 3$)$ and 5) is satisfied, i.e., char $k \nmid\left(\frac{n-1}{2}\right)$, then $\varepsilon_{1} h_{s-1}$ generates $\mathrm{HH}^{s}(R)$ because $\varepsilon_{1} h_{s-1} \neq 0$ by Lemma 1. If the assumption of one of items 4) and 6) is satisfied, then $\xi_{s}$ generates $\mathrm{HH}^{s}(R)$, because $\xi_{s} \neq 0$ by Lemma 1 ,

9. Assume that $s=(l+1)(2 n-3)-1$ satisfies condition (2.6). Then the elements $f_{s}$ and $p_{s}$ are defined, and the assumption of one of items 1) and 2) is satisfied. Since $f_{s}$ and $p_{s}$ are linearly independent over $k$ by Corollary 1 and $\operatorname{dim}_{k} \operatorname{HH}^{s}(R)=2$, it follows that $f_{s}$ and $p_{s}$ generate $\mathrm{HH}^{s}(R)$.

10. Assume that $s=l(2 n-3)$ satisfies assumption (2.6). Then the elements $f_{s-1}$, $p_{s-1}, \chi_{s}$, and $\chi_{s}^{\prime}$ are defined, and the assumption of one of items 1) and 2) is satisfied. We have $\operatorname{dim}_{k} \mathrm{HH}^{s}(R)=2$ in this case. Since $\chi_{s}$ and $\chi_{s}^{\prime}$ are linearly independent over $k$ by Lemma 1, they generate $\mathrm{HH}^{s}(R)$. If the assumption of item 1) is satisfied, i.e., char $k \nmid n-1$, then $\chi_{s}$ and $\chi_{s}^{\prime}$ are expressed in terms of $\varepsilon_{1} f_{s-1}$ and $\varepsilon_{1} p_{s-1}$ (see (4.2)). Thus, $\varepsilon_{1} f_{s-1}$ and $\varepsilon_{1} p_{s-1}$ generate $\mathrm{HH}^{s}(R)$. If the assumption of item 2) is satisfied, then $\varepsilon_{1} f_{s-1}$ and $\chi_{s}$ generate $\mathrm{HH}^{s}(R)$, because $\varepsilon_{1} f_{s-1}=\chi_{s}-\chi_{s}^{\prime}$ by formula (4.2).

The claims in items 1$)-6$ ) of the proposition are proved. 
b) Assume that $r=1$. We consider the case where $s=0$. Then $\operatorname{HH}^{0}(R)=\operatorname{Ker} \delta^{0}$ and $\operatorname{dim}_{k} \mathrm{HH}^{0}(R)=n+1$. Since the elements $f_{0}, \varepsilon_{0}^{(j)}(1 \leq j \leq n)$ are linearly independent over $k$ and lie in $\operatorname{Ker} \delta^{0}$, they generate $\operatorname{HH}^{0}(R)$.

The cases where $s \geq 1$ satisfies one of conditions (2.7)-(2.15) in Theorem 3 are analyzed much as in part a) of this proof, with the use of Remark 5 .

We have shown that $\mathrm{HH}^{s}(R) \subset \mathcal{H}$ for each of cases 1)-7). Consequently, $\mathrm{HH}^{*}(R)=\mathcal{H}$. The proposition is proved.

Corollary 2. In all cases 1)-7) of Proposition 2, the algebra $\mathrm{HH}^{*}(R)$ is generated by the elements of the corresponding generating set whose degree does not exceed $\lambda$ (see (2.1)).

Proof. This follows from Proposition 2 and Remark 3.

\section{$\S 5$. Relations}

In this section, we find several relations satisfied by the elements of $\operatorname{HH}^{*}(R)$ introduced in the preceding two sections.

First, we produce formulas for the products of the form $x y$, where $y \in\left\{f_{s}, g_{s}, h_{s}, p_{s}\right\}$.

Remark 6. In the sequel, we assume that either $s_{i}=2 m_{i}+l_{i}(2 n-3)$ or $s_{i}=2 m_{i}+1+$ $l_{i}(2 n-3)$, where $0 \leq s_{i}-l_{i}(2 n-3) \leq 2 n-4$. In each specific case, it will be clear which version occurs, because of the other assumptions about $s_{i}$ (cf. Remark 2).

Proposition 3. The following identities are valid in the algebra $\mathrm{HH}^{*}(R)$ :

$$
\begin{aligned}
& \left.\begin{array}{c}
f_{s_{1}} f_{s_{2}}=\left\{\begin{array}{ll}
f_{s_{1}+s_{2}} & \text { if } m_{1}+m_{2} \leq n-2, \\
2(-1)^{n-1} g_{s_{1}+s_{2}} & \text { if } m_{1}+m_{2} \geq n-1 ;
\end{array}\right\} \\
\varepsilon_{1} f_{s_{1}}=0 \text { if } s_{1}=(l+1)(2 n-3)-1,2 \nmid n ;
\end{array}\right\} \\
& g_{s_{1}} f_{s_{2}}=\left\{\begin{array}{ll}
g_{s_{1}+s_{2}} & \text { if } m_{1}+m_{2} \leq n-3, \\
\xi_{s_{1}+s_{2}} & \text { if } m_{1}+m_{2}=n-2, \\
0 & \text { if } m_{1}+m_{2} \geq n-1 ;
\end{array}\right\} \\
& \left.\begin{array}{l}
p_{s_{1}} f_{s_{2}}= \begin{cases}-g_{s_{1}+s_{2}} & \text { if } m_{2} \geq 1 \\
p_{s_{1}+s_{2}} & \text { if } m_{2}=0 ;\end{cases} \\
p_{s_{1}} g_{s_{2}}=0 ; \quad p_{s_{1}} p_{s_{2}}=-\left(\frac{n}{2}\right) g_{s_{1}+s_{2}} ;
\end{array}\right\} \\
& h_{s_{1}} f_{s_{2}}= \begin{cases}0 & \text { if } m_{2} \geq 1 \text { and char } k \neq 2, \\
\varepsilon_{1} f_{s_{1}+s_{2}-1} & \text { if } m_{2} \geq 1 \text { and char } k=2, \\
h_{s_{1}+s_{2}} & \text { if } m_{2}=0\end{cases} \\
& h_{s_{1}} g_{s_{2}}= \begin{cases}0 & \text { if } \operatorname{char} k \neq 2, \\
\varepsilon_{1} g_{s_{1}+s_{2}-1} & \text { if } m_{2} \geq 1 \text { and char } k=2, \\
\chi_{s_{1}+s_{2}} & \text { if } m_{2}=0 \text { and char } k=2 ;\end{cases} \\
& h_{s_{1}} h_{s_{2}}=-\left(\frac{n-1}{2}\right) g_{s_{1}+s_{2}} \text {; }
\end{aligned}
$$

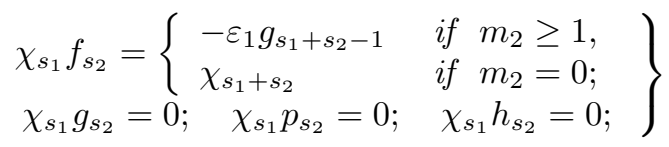

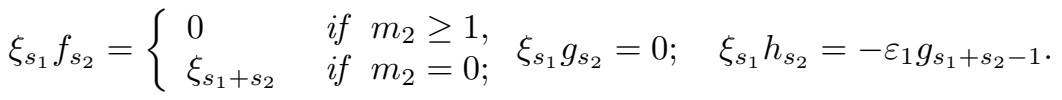

Proof. If the elements $f_{s_{1}}$ and $f_{s_{2}}$ are defined and $m_{1}+m_{2} \leq n-2$, then it is easy to check that the element $f_{s}$ is defined, where $s=s_{1}+s_{2}$ (and moreover, we have $m=m_{1}+m_{2}$ and $l=l_{1}+l_{2}$ ). Using (3.1) and formulas in items 1.1) and 1.5) of Proposition 1, we 
obtain $f_{s_{1}} T_{s_{1}}\left(f_{s_{2}}\right)=f_{s}$. If $m_{1}+m_{2} \geq n-1$, then the element $g_{s}$ is defined, where $s=s_{1}+s_{2}$ (and moreover, we have $m=m_{1}+m_{2}-(n-1)$ and $\left.l=l_{1}+l_{2}+1\right)$. Again, using (3.1) and formulas in items 1.1) and 1.5) of Proposition 1, we see that

$$
f_{s_{1}} T_{s_{1}}\left(f_{s_{2}}\right)=(-1)^{n-1} \sum_{i=1}^{r}\left(2 \sum_{j=n-1-m}^{n-2} t_{i, m, j}+u_{i, m, n-1}-u_{i, m, n}+v_{i, m, n-1}-v_{i, m, n}\right) \text {. }
$$

Relation (3.2) shows that we need to prove that the last expression equals

$$
2(-1)^{n-1} \sum_{i=1}^{r}\left(\sum_{j=n-1-m}^{n-2} t_{i, m, j}+u_{i, m, n-1}+v_{i, m, n-1}\right),
$$

or equivalently, that $v=\sum_{i=1}^{r}\left(u_{i, m, n-1}+u_{i, m, n}+v_{i, m, n-1}+v_{i, m, n}\right)=0$ in $\operatorname{HH}^{*}(R)$. Put $w=\sum_{i=1}^{r} \sum_{j=1}^{n-2-m} w_{i, m, j} \in \operatorname{Hom}_{\Lambda}\left(Q_{s-1}, R\right)$. It is easily seen that $\delta^{s-1}(w)=v$. Now we assume that $s_{1}=(l+1)(2 n-3)-1$ and $2 \nmid n$ and that the element $f_{s_{1}}$ is defined. In this case, we have char $k=2$. Using formulas of items 1.3) and 1.5) of Proposition 1 and the definition of $\varepsilon_{1}$, we obtain $\varepsilon_{1} T_{1}\left(f_{s_{1}}\right)=v_{r, n-1}+v_{r, n}$. Hence, using (4.4) and the fact that char $k=2$, we get $v_{r, n-1}+v_{r, n}=0$. This proves identities (5.1).

If the elements $g_{s_{1}}$ and $f_{s_{2}}$ are defined and $m_{1}+m_{2} \leq n-3$, then it is easy to check that the element $g_{s}$ is defined, where $s=s_{1}+s_{2}$ (and moreover, we have $m=m_{1}+m_{2}$ and $l=l_{1}+l_{2}$ ). Using (3.2) and formulas of items 1.2) and 1.5) of Proposition 1, we obtain $g_{s_{1}} T_{s_{1}}\left(f_{s_{2}}\right)=g_{s}$. If $m_{1}+m_{2} \geq n-1$, then it is easily seen that $s_{1}+s_{2}$ satisfies none of the conditions in Theorems 2 and 3 i.e., we have $\operatorname{dim}_{k} \mathrm{HH}^{s_{1}+s_{2}}(R)=0$. Consequently, $g_{s_{1}} f_{s_{2}}=0$ in $\mathrm{HH}^{*}(R)$. Now we assume $m_{1}+m_{2}=n-2$. Then the element $\xi_{s}$ is defined, where $s=s_{1}+s_{2}$ (and moreover, we have $l=l_{1}+l_{2}+1$ ). Suppose that char $k \neq 2$. Then $2 \nmid l_{1}, 2 \mid l_{2}$. Since $2 \nmid m_{1}+l_{1} n$ and $2 \mid m_{2}+l_{2} n$, we have $2 \nmid m_{1}-n$ and $2 \mid m_{2}$, i.e., $2 \nmid m_{1}-n+m_{2}=0$. We arrive at a contradiction, whence we conclude that char $k=2$.

Using (3.2) and formulas of parts 1.3) and 1.5) of Proposition 1, we obtain

$$
g_{s_{1}} T_{s_{1}}\left(f_{s_{2}}\right)=\sum_{i=1}^{r} \sum_{j=1}^{n-2} t_{i, n-2, j}
$$

Since $\xi_{s}=t_{r, n-2,1}$, we need to prove that $\sum_{i=1}^{r} \sum_{j=1}^{n-2} t_{i, n-2, j}-t_{r, n-2,1} \in \operatorname{Im} \delta_{s-1}$. From (4.3) we know that this fact is equivalent to the relation $r(n-2)-1=0$ (in $k$ ), or is equivalent to the fact that $2 \nmid r$ and $2 \nmid n$. Since $2 \nmid \ln$, we have $2 \nmid n$. Since $2 \nmid l(n-1)-1$ and $r \mid l(n-1)-1$, we obtain $2 \nmid r$. Assume that the elements $g_{s_{1}}$ and $g_{s_{2}}$ are defined. Then we get $g_{s_{1}} T_{s_{1}}\left(g_{s_{2}}\right)=0$ by analyzing successively the cases where $m_{1}+m_{2} \leq n-3$ or $m_{1}+m_{2} \geq n-2$, and by using (3.2) and formulas of items 2.2), 2.5), and 2.6) of Proposition 10. Consequently, identities (5.2) are proved.

Assume that the elements $p_{s_{1}}$ and $f_{s_{2}}$ are defined and $m_{2} \geq 1$. Then it is easily seen that the element $g_{s}$ is defined, where $s=s_{1}+s_{2}$ (and moreover, we have $m=m_{2}-1$ and $\left.l=l_{1}+l_{2}+1\right)$. Now we obtain

$$
p_{s_{1}} T_{s_{1}}\left(f_{s_{2}}\right)=\sum_{i=1}^{r}\left(\sum_{j=1}^{n-3-m}(-1)^{j+1}\left(w_{i, m+1, j}+u_{i, m, n-1}+u_{i, m, n}\right)\right)-g_{s}
$$

with the help of (3.4) and formulas of items 1.4) and 1.5) of Proposition 1. Consequently, it suffices to prove that $w_{i, m+1, j}+u_{i, m, n-1}+u_{i, m, n}=0$ in $\operatorname{HH}^{*}(R)$ for $1 \leq i \leq r$ and $1 \leq j \leq n-3-m$. It is easy to check that

$$
\delta^{s-1}\left(\sum_{z=j+1}^{n-2-m} w_{i, m, z}\right)=w_{i, m+1, j}+u_{i, m, n-1}+u_{i, m, n}
$$


If $m_{2}=0$, then the element $p_{s}$ is defined, where $s=s_{1}+s_{2}$ (and moreover, we have $\left.l=l_{1}+l_{2}\right)$. Now we can get the relation $p_{s_{1}} T_{s_{1}}\left(f_{s_{2}}\right)=p_{s}$ with the help of (3.4) and formulas of 1.1) and 1.5) of Proposition 1. If the elements $p_{s_{1}}$ and $g_{s_{2}}$ are defined, then, as above, $s_{1}+s_{2}$ satisfies none of the conditions of Theorems 2 and 3 , so that $\operatorname{dim}_{k} \mathrm{HH}^{s_{1}+s_{2}}(R)=0$. Consequently, $p_{s_{1}} g_{s_{2}}=0$ in $\mathrm{HH}^{*}(R)$. Assume that the elements $p_{s_{1}}$ and $p_{s_{2}}$ are defined. Then the element $g_{s}$ is defined, where $s=s_{1}+s_{2}$ (and moreover, $m=n-3$ and $\left.l=l_{1}+l_{2}+1\right)$. Using formulas of 4.2) and 4.6) of Proposition 1 and formulas (3.2) and (3.4), we obtain $p_{s_{1}} T_{s_{1}}\left(p_{s_{2}}\right)=-\left(\frac{n}{2}\right) g_{s}$. This completes the proof of $(5.3)$.

Assume that the elements $h_{s_{1}}$ and $f_{s_{2}}$ are defined and $m_{2} \geq 1$. If char $k \neq 2$, then $s_{1}+s_{2}$ satisfies none of the conditions of Theorems 2 and 3, whence $\operatorname{dim}_{k} \mathrm{HH}^{s_{1}+s_{2}}(R)=0$. Consequently, $h_{s_{1}} f_{s_{2}}=0$ in $\mathrm{HH}^{*}(R)$. Now, assume char $k=2$. Then the element $f_{s-1}$ is defined, where $s=s_{1}+s_{2}$ (and moreover, $m=m_{2}-1$ and $l=l_{1}+l_{2}+1$ ). The identity

$$
h_{s_{1}} T_{s_{1}}\left(f_{s_{2}}\right)=\sum_{i=1}^{r}\left(\sum_{j=1}^{n-3-m} w_{i, m+1, j}+(m+1)\left(u_{i, m, n-1}+u_{i, m, n}\right)\right)
$$

is verified by using (3.3) and formulas of items 1.4) and 1.5) of Proposition 11. In the proof of part 1) of Lemma 1 we saw that $\varepsilon_{1} T_{1}\left(f_{s-1}\right)=u_{1, m, n-1}+u_{1, m, n}$. Hence, we need to prove that in $\mathrm{HH}^{*}(R)$,

$$
v=\sum_{i=1}^{r}\left(\sum_{j=1}^{n-3-m} w_{i, m+1, j}+(m+1)\left(u_{i, m, n-1}+u_{i, m, n}\right)\right)-\left(u_{1, m, n-1}+u_{1, m, n}\right)=0 .
$$

We define

$$
\begin{aligned}
w & =\sum_{j=1}^{n-2-m} j w_{1, m, j}+\left(u_{r, n-1}+u_{r, n}\right) \\
& \quad+\sum_{i=2}^{r}\left(\sum_{j=1}^{n-2-m}(i+j) w_{i, m, j}+(i+1)\left(u_{i-1, n-1}+u_{i-1, n}\right)\right) \\
& \in \operatorname{Hom}_{\Lambda}\left(Q_{s-1}, R\right) .
\end{aligned}
$$

Since $2 \nmid n$ and $2 \nmid r$, it is easy to check that $\delta^{s-1}(w)=v$. If $m_{2}=0$, then the element $h_{s}$ is defined, where $s=s_{1}+s_{2}$ (and moreover, $l=l_{1}+l_{2}$ ). Then we can obtain $h_{s_{1}} T_{s_{1}}\left(f_{s_{2}}\right)=h_{s}$ by using (3.3) and formulas of items 1.1) and 1.5) of Proposition 1 . Now, assume that the elements $h_{s_{1}}$ and $g_{s_{2}}$ are defined. Assume also that char $k \neq 2$. It is easily seen that if $\operatorname{dim}_{k} \mathrm{HH}^{s}(R)>0$, then, among the conditions of Theorems 2 and 3 , only (2.11) may be fulfilled (and moreover, we have $m=m_{2}$ and $l=l_{1}+l_{2}+1$ ). Since $2 \mid l$ in this case, we see that $\operatorname{HH}^{s}(R)$ is generated by $f_{s}$. We have $h_{s_{1}} g_{s_{2}}-\varkappa f_{s}=0$ in $\mathrm{HH}^{*}(R)$ for some $\varkappa \in k$. Clearly, $h_{s_{1}} T_{s_{1}}\left(g_{s_{2}}\right)\left(Q_{s}\right) \subset \operatorname{Rad} R$. If $\varkappa \neq 0$, then we obtain $h_{s_{1}} T_{s_{1}}\left(g_{s_{2}}\right)-\varkappa f_{s} \not \subset \operatorname{Rad} R$, a contradiction. Hence, $\varkappa=0$, whence $h_{s_{1}} g_{s_{2}}=0$ in $\operatorname{HH}^{*}(R)$. Now we assume that char $k=2$ and $m_{2} \geq 1$. Then the element $g_{s-1}$ is defined, where $s=s_{1}+s_{2}$ (and moreover, $m=m_{2}-1$ and $l=l_{1}+l_{2}+1$ ). Using (3.3) and formulas in 2.4) and 2.6) of Proposition 1, we conclude that

$$
h_{s_{1}} T_{s_{1}}\left(g_{s_{2}}\right)=\sum_{i=1}^{r}\left(\sum_{j=n-2-m}^{n-2}\left(t_{i, m+1, j}+t_{i, m+1, n-2-m}\right)+v_{i, n-1}\right) .
$$

Since $\varepsilon_{1} T_{1}\left(g_{s-1}\right)=v_{r, n-1}$, it suffices to prove that $t_{i, m+1, j}+t_{i, m+1, n-2-m}=0$ for $1 \leq i \leq r$ and $n-2-m \leq j \leq n-2$, and that $\sum_{i=1}^{r-1} v_{i, n-1}=0$ in $\operatorname{HH}^{*}(R)$. It is easy 
to verity that

$$
\delta^{s-1}\left(\sum_{z=n-1-m}^{j} t_{i, m, z}\right)=t_{i, m+1, j}+t_{i, m+1, n-2-m}
$$

and

$$
\delta^{s-1}\left(u_{i, m, n-1}+\sum_{z=n-1-m}^{n-2} t_{i, m, z}+v_{i, m, n-1}\right)=v_{i-1, n-1}+v_{i, n} .
$$

The existence of the element $h_{s_{1}}$ implies $2 \nmid r$, whence we have

$$
\sum_{i=1}^{r-1} v_{i, n-1}=\sum_{i=1}^{\frac{r-1}{2}}\left(v_{2 i-1, n-1}+v_{2 i, n-1}\right)=0
$$

in $\mathrm{HH}^{*}(R)$.

Now we assume that char $k=2$ and $m_{2}=0$. Then the element $\chi_{s}$ is defined, where $s=s_{1}+s_{2}$ (and moreover, we have $\left.l=l_{1}+l_{2}+1\right)$. Using (3.3) and formulas of items 2.3) and 2.6) of Proposition 1, we obtain $h_{s_{1}} T_{s_{1}}\left(g_{s_{2}}\right)=\sum_{i=1}^{r} v_{i, n-1}$. Since $\chi_{s}=v_{r, n}$, it suffices to prove that $\sum_{i=1}^{r} v_{i, n-1}-v_{r, n}=0$ in $\mathrm{HH}^{*}(R)$. By (4.4), the last relation is equivalent to the fact that the identity $\sum_{i=1}^{r} 1-1=r-1=0$ is valid in $k$. But we have $2 \nmid r$, so that this is true indeed. Now we assume that the elements $h_{s_{1}}$ and $h_{s_{2}}$ are defined. Then the element $g_{s}$ is defined, where $s=s_{1}+s_{2}$ (and moreover, $m=n-3$ and $\left.l=l_{1}+l_{2}+1\right)$. Using formulas in 3.3) and 3.6) of Proposition 1 and formulas (3.2) and (3.3), we see that $h_{s_{1}} T_{s_{1}}\left(h_{s_{2}}\right)=-\left(\frac{n-1}{2}\right) g_{s}$. Consequently, identities (5.4) are proved.

If the elements $\chi_{s_{1}}$ and $f_{s_{2}}$ are defined and $m_{2} \geq 1$, then it is easy to check that the element $g_{s-1}$ is defined, where $s=s_{1}+s_{2}$ (moreover, we have $m=m_{2}$ and $l=l_{1}+l_{2}$ ). With the help of formulas of items 1.1) and 1.5) of Proposition 1 and using the definition of $\chi_{s_{1}}$, we obtain $\chi_{s_{1}} T_{s_{1}}\left(f_{s_{2}}\right)=v_{r, n}$. From the proof of the part 2) of Lemma 11, we know that $\varepsilon_{1} T_{1}\left(g_{s-1}\right)=v_{r, n-1}$. Because of this we need to prove that $v_{r, n-1}+v_{r, n}=0$ in $\mathrm{HH}^{*}(R)$. It is easily seen that $\delta^{s-1}\left(u_{1, m-1, n-1}+u_{1, m-1, n}\right)=v_{r, n-1}+v_{r, n}$. If $m_{2}=0$, then the element $\chi_{s}$ is defined, where $s=s_{1}+s_{2}$ (moreover, we have $l=l_{1}+l_{2}$ ). Hence, using formulas of parts 1.1) and 1.5) of Proposition 1 and the definition of $\chi_{s_{1}}$, we obtain $\chi_{s_{1}} T_{s_{1}}\left(f_{s_{2}}\right)=\chi_{s}$. Assume that the element $\chi_{s_{1}}$ is defined. If, moreover, the element $g_{s_{2}}$ (respectively, either $h_{s_{2}}$ or $p_{s_{2}}$ ) is defined, then, using formulas of items 2.1), 2.6), 3.1), 3.6), 4.1), and 4.6) of Proposition 1 and the definition of $\chi_{s_{1}}$, we obtain $\chi_{s_{1}} T_{s_{1}}\left(g_{s_{2}}\right)=0$ (respectively, $\chi_{s_{1}} T_{s_{1}}\left(h_{s_{2}}\right)=0$ or $\chi_{s_{1}} T_{s_{1}}\left(p_{s_{2}}\right)=0$ ). This proves identities (5.5) .

Assume that the elements $\xi_{s_{1}}$ and $f_{s_{2}}$ are defined. If we assume additionally that $m_{2} \geq 1$, then, using formulas of 1.1) and 1.5) of Proposition 1 and the definition of $\xi_{s_{1}}$, we obtain $\xi_{s_{1}} T_{s_{1}}\left(f_{s_{2}}\right)=0$. If $m_{2}=0$, then the element $\xi_{s}$ is defined, where $s=$ $s_{1}+s_{2}$ (moreover, $l=l_{1}+l_{2}$ ). Using formulas of parts 1.1) and 1.5) of Proposition 1 and the definition of $\xi_{s_{1}}$, we get $\xi_{s_{1}} T_{s_{1}}\left(f_{s_{2}}\right)=\xi_{s}$. If the elements $\xi_{s_{1}}$ and $g_{s_{2}}$ are defined, then the formulas of items 2.1) and 2.6) of Proposition 11 and the definition of $\xi_{s_{1}}$, yield $\xi_{s_{1}} T_{s_{1}}\left(g_{s_{2}}\right)=0$. If the elements $\xi_{s_{1}}$ and $h_{s_{2}}$ are defined, then it is easily seen that the element $g_{s-1}$ is defined, where $s=s_{1}+s_{2}$ (moreover, $m=n-2$ and $l=l_{1}+l_{2}$ ). Using formulas of items 3.1) and 3.6) of Proposition 1 and the definition of $\xi_{s_{1}}$, we get $\xi_{s_{1}} T_{s_{1}}\left(h_{s_{2}}\right)=-t_{r, n-2,1}$. From the proof of Lemma 1 we know that $\varepsilon_{1} T_{1}\left(g_{s-1}\right)=v_{r, n-1}$. Hence, we need to prove that $t_{r, n-2,1}-v_{r, n-1}=0$ in $\operatorname{HH}^{*}(R)$. We put $w=\sum_{j=2}^{n-2} t_{r, n-3, j}+v_{r, n-3, n-1} \in \operatorname{Hom}_{\Lambda}\left(Q_{s-1}, R\right)$. Then it is easy to verify that $\delta^{s-1}(w)=t_{r, n-2,1}-v_{r, n-1}$, i.e., $t_{r, n-2,1}-v_{r, n-1}=0$ in $\operatorname{HH}^{*}(R)$. This completes the proof of (5.6).

Proposition 4. The product of any two elements of the set $\left\{\varepsilon_{1}, \chi_{s}, \xi_{s}\right\}$ is equal to 0 in $\mathrm{HH}^{*}(R)$. 
Proof. Theorems 2 and 3 show that $\operatorname{HH}^{2}(R)=0$, whence $\left(\varepsilon_{1}\right)^{2}=0$. Assume that the element $\xi_{s}$ is defined. By Theorems 2 and 3. $\mathrm{HH}^{s+1}(R)$ can be nonzero only if $s+1$ satisfies condition (2.9). Then it is easily seen that $\mathrm{HH}^{s+1}(R)$ is generated by $g_{s+1}$. If $\varepsilon_{1} \xi_{s} \neq 0$, then $\varepsilon_{1} \xi_{s}=\varkappa g_{s+1}$ with $\varkappa \in k$. Multiplying this by $\varepsilon_{1}$, we obtain $0=\varkappa \varepsilon_{1} g_{s+1}$. By Lemma 1 we have $\varepsilon_{1} g_{s+1} \neq 0$, whence $\varkappa=0$.

Assume that the element $\chi_{s}$ is defined. Then, using Theorems 2 and 3 , we easily check that $\mathrm{HH}^{s+1}(R)$ can be nonzero only if $s+1$ satisfies condition (2.10). It is easily seen that $\mathrm{HH}^{s+1}(R)$ is generated by the element $\varepsilon_{1} f_{s}$ in this case. Note that the elements $f_{4}$ and $f_{s+4}$ are defined. If we assume, moreover, that $2 \nmid n$, then $2 n-3 \nmid s+5$, because $2 n-3 \mid s$ and $n \geq 5$. Consequently, $\varepsilon_{1} f_{s+4} \neq 0$ by Lemma1 If $2 \mid n$, then $\varepsilon_{1} f_{s+4} \neq 0$ by Lemma 11 and Remark 4. Furthermore, we have $\varepsilon_{1} \chi_{s}=\varkappa \varepsilon_{1} f_{s}$ with $\varkappa \in k$. Multiplying this by $f_{4}$, we obtain $0=-\left(\varepsilon_{1}\right)^{2} g_{s+3}=\varkappa \varepsilon_{1} f_{s+4}$. Since $\varepsilon_{1} f_{s+4} \neq 0$, we have $\varkappa=0$.

Assume that the elements $\chi_{s_{1}}$ and $\chi_{s_{2}}$ are defined and suppose that $2 \nmid n$. It is easily seen that $\mathrm{HH}^{s}(R)$ with $s=s_{1}+s_{2}$ is generated by the elements of the set $\left\{f_{s}, \chi_{s}\right\}$ that are defined for $s$. Then we have $\chi_{s_{1}} \chi_{s_{2}}=\varkappa_{1} f_{s}+\varkappa_{2} \chi_{s}$ with $\varkappa_{1}, \varkappa_{2} \in k$ (here, if an element is not defined for $s$, we assume that the corresponding $\varkappa_{i}$ is zero). Multiplying the last identity by $\varepsilon_{1}$, we obtain $0=\varkappa_{1} \varepsilon_{1} f_{s}$. Since $\varepsilon_{1} f_{s} \neq 0$, we have $\varkappa_{1}=0$. Multiplying the same identity by $f_{s_{1}-1}$, we get $0=-\chi_{s_{1}} \varepsilon_{1} g_{s-2}=-\varkappa_{2} \varepsilon_{1} g_{s_{1}+s-2}$, using the graded commutativity of the algebra $\mathrm{HH}^{*}(R)$. Consequently, $\varkappa_{2}=0$.

Again, assume that the elements $\chi_{s_{1}}$ and $\chi_{s_{2}}$ are defined and that $2 \mid n$. It is easily seen that $\mathrm{HH}^{s}(R)$ with $s=s_{1}+s_{2}$ is generated by the elements of the set $\left\{f_{s}, \chi_{s}, \varepsilon_{1} f_{s-1}\right\}$ that are defined for $s$. Then $\chi_{s_{1}} \chi_{s_{2}}=\varkappa_{1} f_{s}+\varkappa_{2} \chi_{s}+\varkappa_{3} \varepsilon_{1} f_{s-1}$ with $\varkappa_{1}, \varkappa_{2}, \varkappa_{3} \in k$ (here, if an element is not defined for $s$, we assume that the corresponding $\varkappa_{i}$ is zero). Multiplying the last identity by $\varepsilon_{1}$, once again we see that $\varkappa_{1}=0$. Multiplying the resulting relation by $p_{s_{1}-1}$ and using the graded commutativity of the algebra $\mathrm{HH}^{*}(R)$, we get $0=-\varkappa_{3} \varepsilon_{1} g_{s_{1}+s-2}$. Consequently, $\varkappa_{3}=0$. Now we multiply the identity obtained by $f_{s_{1}-1}$ to show that $\varkappa_{2}=0$.

Assume that the elements $\xi_{s_{1}}$ and $\xi_{s_{2}}$ are defined. It is easily seen that $\operatorname{HH}^{s}(R)$ with $s=s_{1}+s_{2}$ is generated by the elements of the set $\left\{f_{s}, \chi_{s}\right\}$ that are defined for $s$. Then $\xi_{s_{1}} \xi_{s_{2}}=\varkappa_{1} f_{s}+\varkappa_{2} \chi_{s}$ with $\varkappa_{1}, \varkappa_{2} \in k$ (here, if an element is not defined for $s$, then we assume that the corresponding $\varkappa_{i}$ is zero). Multiplying this identity by $\varepsilon_{1}$ and then, in the case where $\chi_{s}$ is defined, by $f_{s-1}$, we see that $\varkappa_{1}=0$, implying $\varkappa_{2}=0$. Finally, we consider the last possibility, namely, we assume that the elements $\xi_{s_{1}}$ and $\chi_{s_{2}}$ are defined. Theorems 2 and 3 show that $\mathrm{HH}^{s}(R)$ can be nonzero for $s=s_{1}+s_{2}$ only if $s$ satisfies condition (2.8). It is easily seen that $\mathrm{HH}^{s}(R)$ is generated by $\xi_{s}$ in this case. Then $\xi_{s_{1}} \chi_{s_{2}}=\varkappa \xi_{s}$ with $\varkappa \in k$. Multiplying this by $h_{s_{1}-1}$, we obtain $0=-\varkappa \varepsilon_{1} g_{s-2}$, whence $\varkappa=0$. Proposition 4 is proved, in view of the graded commutativity of the algebra $\mathrm{HH}^{*}(R)$.

Corollary 3. The algebra $\mathrm{HH}^{*}(R)$ is commutative.

Proof. Since $\mathrm{HH}^{*}(R)$ is graded commutative, it is commutative in the case where char $k=$ 2. Assume char $k \neq 2$. Then, by graded commutativity, it suffices to prove that the product of any two different generators of odd degree is 0 . Since char $k \neq 2$, it is easily seen that the elements $f_{s}, g_{s}, h_{s}$, and $p_{s}$ are defined only for even $s$. Hence, all generators of odd degree are among the elements $\varepsilon_{1}, \chi_{s}$, and $\xi_{s}$. Now, the desired statement follows from Proposition 4

It remains to compute the products of the elements $\varepsilon_{0}^{(j)}(1 \leq j \leq n)$ by themselves and by other elements. Since the elements $\varepsilon_{0}^{(j)}$ are defined only for $r=1$, we assume that $r=1$ in the remaining part of this section. 
Proposition 5. 1) We have $\varepsilon_{0}^{(i)} \varepsilon_{0}^{(j)}=0$ for $1 \leq i, j \leq n$.

2.1) If char $k \neq 2$, then $\varepsilon_{0}^{(j)} \mathrm{HH}^{s}(R)=0$ for $s \geq 1$ and $1 \leq j \leq n$.

2.2) Assume that char $k=2$ and $2 \nmid n$. Then $\varepsilon_{0}^{(j)} \mathrm{HH}^{s}(R)=0$ for $s \geq 1$ and $1 \leq j \leq n-2$. Moreover, for $q \in\{n-1, n\}$ we have

$$
\begin{aligned}
& \varepsilon_{0}^{(q)} f_{s}=\left\{\begin{array}{ll}
\varepsilon_{1} g_{s-1} & \text { if } m \geq 1, \\
\chi_{s} & \text { if } m=0
\end{array} \quad(s \geq 1)\right. \\
& \varepsilon_{0}^{(q)} g_{s}=\varepsilon_{0}^{(q)} h_{s}=\varepsilon_{0}^{(q)} \chi_{s}=\varepsilon_{0}^{(q)} \xi_{s}=\varepsilon_{0}^{(q)} \varepsilon_{1}=0 .
\end{aligned}
$$

2.3) Assume that char $k=2$ and $2 \mid n$. Then

$$
\begin{aligned}
& \varepsilon_{0}^{(j)} f_{s}=\left\{\begin{array}{ll}
0 & \text { if } m \geq 1 \text { and } 1 \leq j \leq n-2, \\
\varepsilon_{1} g_{s-1} & \text { if } m \geq 1 \text { and } j \in\{n-1, n\}, \\
\chi_{s}+\chi_{s}^{\prime} & \text { if } m=0 \text { and } 1 \leq j \leq n-2 \\
\chi_{s}^{\prime} & \text { if } m=0 \text { and } j=n-1, \\
\chi_{s} & \text { if } m=0 \text { and } j=n,
\end{array} \quad(s \geq 1) ;\right. \\
& \varepsilon_{0}^{(j)} p_{s}= \begin{cases}\varepsilon_{1} g_{s-1} & \text { if } 1 \leq j \leq n-1, \\
0 & \text { if } j=n ;\end{cases} \\
& \varepsilon_{0}^{(j)} g_{s}=\varepsilon_{0}^{(j)} \chi_{s}=\varepsilon_{0}^{(j)} \varepsilon_{1}=0 \quad(1 \leq j \leq n) .
\end{aligned}
$$

Proof. 1) Since $\varepsilon_{0}^{(j)}\left(Q_{0}\right) \subset \operatorname{Rad} R$, we have $T_{0}\left(\varepsilon_{0}^{(j)}\right)\left(Q_{0}\right) \subset \operatorname{Rad} Q_{0}$ (because $\mu(\theta) \notin \operatorname{Rad} R$ for $\left.\theta \notin \operatorname{Rad} Q_{0}\right)$. Obviously, $\varepsilon_{0}^{(i)}(\theta)=0$ for $\theta \in \operatorname{Rad} Q_{0}$. Consequently, $\varepsilon_{0}^{(i)} \varepsilon_{0}^{(j)}=0$.

2) Observe the following fact. Assume that $\theta \in \mathrm{HH}^{s}(R)$ with $s \geq 1$, and suppose $\varepsilon_{0}^{(j)} f_{\lambda} \theta=\omega f_{\lambda}$. Then the relation $\varepsilon_{0}^{(j)} \theta=\omega$ follows from Remark 3 and the commutativity of $\mathrm{HH}^{*}(R)$.

2.1) If char $k \neq 2$, then Theorem [3] shows that $\operatorname{dim}_{k} \mathrm{HH}^{\lambda}(R)=1$, i.e., $\mathrm{HH}^{\lambda}(R)$ is generated by the element $f_{\lambda}$. Since $\varepsilon_{0}^{(j)}\left(Q_{0}\right) \subset \operatorname{Rad} R$ and $f_{\lambda}$ is an isomorphism, we have $\varepsilon_{0}^{(j)} f_{\lambda}=0$. Then item 2.1) of Proposition 5 follows from the above observation.

2.2) The relations $\varepsilon_{0}^{(j)} f_{\lambda}=t_{n-2, j}$ for $1 \leq j \leq n-2$ and $\varepsilon_{0}^{(q)} f_{\lambda}=v_{q}$ for $q \in\{n-1, n\}$ are obtained by using formulas of items 1.1) and 1.5) of Proposition 11 (as before, in our notation we omit the first index, which is always equal to 1 for $r=1$ ).

Now, suppose that char $k=2$ and $2 \nmid n$. It is easily seen that $\operatorname{HH}^{\lambda}(R)$ is generated by the elements $f_{\lambda}$ and $\chi_{\lambda}$. From the proof of item 5) of Lemma 1, it follows that $t_{n-2, j}=0$ for $1 \leq j \leq n-2$ and $v_{n-1}=v_{n}=\chi_{\lambda}$. Then all identities of item 2.2) follow from the observation at the beginning of part 2 of the proof, from Propositions 3 and 4 and from the corollary to Proposition 4 .

2.3) Assume that char $k=2$ and $2 \mid n$. It is easily seen that $\operatorname{HH}^{\lambda}(R)$ is generated by the elements $f_{\lambda}, \chi_{\lambda}$, and $\chi_{\lambda}^{\prime}$. From the proof of part 4) of Lemma 1, it follows that $t_{n-2, j}=\chi_{\lambda}+\chi_{\lambda}^{\prime}$ with $1 \leq j \leq n-2, v_{n-1}=\chi_{\lambda}^{\prime}$, and $v_{n}=\chi_{\lambda}$. Since (4.2) implies $\chi_{s}^{\prime}=\chi_{s}+\varepsilon_{1} f_{s-1}$, all identities in item 2.3) follow from the observation at the beginning of part 2) of the proof, from Propositions 3 and 4 , and from the corollary to Proposition 4 .

\section{§6. MAIN RESULT}

In this section, we describe the algebra $\mathrm{HH}^{*}(R)$ with the help of generators and defining relations.

With each element $\theta \neq f_{0}$ in the set of generators of the algebra $\operatorname{HH}^{*}(R)$, as described in Corollary 2 we associate a variable $\widetilde{\theta}$. 
We consider the following sets (in all cases, a variable with index $s$ is included in this set if this variable is defined):

$$
\mathcal{A}=\left\{\widetilde{\varepsilon}_{1}, \widetilde{f}_{s}, \widetilde{g}_{s}\right\} ; \mathcal{B}_{1}=\left\{\widetilde{p}_{s}\right\} ; \mathcal{B}_{2}=\left\{\widetilde{h}_{s}\right\} ; \quad \mathcal{C}_{1}=\left\{\widetilde{\chi}_{s}\right\} ; \mathcal{C}_{2}=\left\{\widetilde{\xi}_{s}\right\} ; \mathcal{D}=\left\{\widetilde{\varepsilon}_{0}^{(j)}\right\}_{1 \leq j \leq n} .
$$

Next, we define the following sets:

$$
\begin{aligned}
\mathcal{X}_{1} & =\mathcal{A} \cup \mathcal{B}_{1} ; & \mathcal{X}_{2} & =\mathcal{X}_{3}=\mathcal{X}_{1} \cup \mathcal{D} ; \\
\mathcal{X}_{4} & =\mathcal{X}_{1} \cup \mathcal{C}_{1} ; & \mathcal{X}_{5} & =\mathcal{X}_{4} \cup \mathcal{D} ; \\
\mathcal{X}_{6} & =\mathcal{A} \cup \mathcal{B}_{2} ; & \mathcal{X}_{7} & =\mathcal{X}_{6} \cup \mathcal{D} ; \\
\mathcal{X}_{8} & =\mathcal{X}_{6} \cup \mathcal{C}_{2} ; & \mathcal{X}_{9} & =\mathcal{X}_{8} \cup \mathcal{D} ; \\
\mathcal{X}_{10} & =\mathcal{X}_{6} \cup \mathcal{C}_{1} ; & \mathcal{X}_{11} & =\mathcal{X}_{10} \cup \mathcal{D} ; \\
\mathcal{X}_{12} & =\mathcal{X}_{6} \cup \mathcal{C}_{1} \cup \mathcal{C}_{2} ; & \mathcal{X}_{13} & =\mathcal{X}_{12} \cup \mathcal{D} .
\end{aligned}
$$

On $k\left[\mathcal{X}_{i}\right](1 \leq i \leq 13)$, we introduce a grading such that

$$
\begin{array}{rlll}
\operatorname{deg} \widetilde{\varepsilon}_{0}^{(j)}=0, & \operatorname{deg} \widetilde{\varepsilon}_{1}=1, & \operatorname{deg} \tilde{f}_{s}=s, & \operatorname{deg} \widetilde{g}_{s}=s, \\
\operatorname{deg} \tilde{h}_{s}=s, & \operatorname{deg} \widetilde{p}_{s}=s, & \operatorname{deg} \tilde{\chi}_{s}=s, & \operatorname{deg} \widetilde{\xi}_{s}=s .
\end{array}
$$

Let $s=a \lambda+b$ with $a \in \mathbb{N} \cup\{0\}$ and $1 \leq b \leq \lambda$. Then we put

$$
\phi(s)=a ; \quad \psi(s)=b .
$$

Next, let $\mathcal{E}_{\mathcal{A}}$ denote the following set of homogeneous elements of the algebra $k[\mathcal{A}]$ :

$$
\begin{aligned}
\left\{\left(\widetilde{\varepsilon}_{1}\right)^{2}\right\} & \cup\left\{\tilde{f}_{s_{1}} \tilde{f}_{s_{2}}-\tilde{f}_{\psi\left(s_{1}+s_{2}\right)}\left(\tilde{f}_{\lambda}\right)^{\phi\left(s_{1}+s_{2}\right)} \mid m_{1}+m_{2} \leq n-2\right\} \\
& \cup\left\{\tilde{f}_{s_{1}} \tilde{f}_{s_{2}}+2(-1)^{n} \widetilde{g}_{\psi\left(s_{1}+s_{2}\right)}\left(\tilde{f}_{\lambda}\right)^{\phi\left(s_{1}+s_{2}\right)} \mid m_{1}+m_{2} \geq n-1\right\} \\
& \cup\left\{\widetilde{g}_{s_{1}} \tilde{f}_{s_{2}}-\widetilde{g}_{\psi\left(s_{1}+s_{2}\right)}\left(\tilde{f}_{\lambda}\right)^{\phi\left(s_{1}+s_{2}\right)} \mid m_{1}+m_{2} \leq n-3\right\} \\
& \cup\left\{\widetilde{g}_{s_{1}} \tilde{f}_{s_{2}} \mid m_{1}+m_{2} \geq n-1\right\} \cup\left\{\widetilde{g}_{s_{1}} \widetilde{g}_{s_{2}}\right\} .
\end{aligned}
$$

Also, $\mathcal{E}_{\mathcal{D}}$ will be the following set of homogeneous elements of the algebra $k[\mathcal{D}]$ :

$$
\left\{\widetilde{\varepsilon}_{0}^{\left(j_{1}\right)} \widetilde{\varepsilon}_{0}^{\left(j_{2}\right)} \mid 1 \leq j_{1}, j_{2} \leq n\right\} .
$$

We denote by $\mathcal{E}_{\mathcal{A} \cup \mathcal{B}_{2} \cup \mathcal{C}_{1}}$ the following set of homogeneous elements of the algebra $k\left[\mathcal{A} \cup \mathcal{B}_{2} \cup \mathcal{C}_{1}\right]:$

$$
\begin{aligned}
& \left\{\widetilde{\varepsilon}_{1} \tilde{f}_{s} \mid s=(l+1)(2 n-3)-1 \text { and condition (2.4) is satisfied }\right\} \\
& \cup\left\{\widetilde{h}_{s_{1}} \tilde{f}_{s_{2}}-\widetilde{\varepsilon}_{1} \tilde{f}_{\psi\left(s_{1}+s_{2}-1\right)}\left(\tilde{f}_{\lambda}\right)^{\phi\left(s_{1}+s_{2}-1\right)} \mid m_{2} \geq 1\right\} \\
& \cup\left\{\widetilde{h}_{s_{1}} \widetilde{g}_{s_{2}}-\widetilde{\varepsilon}_{1} \widetilde{g}_{\psi\left(s_{1}+s_{2}-1\right)}\left(\tilde{f}_{\lambda}\right)^{\phi\left(s_{1}+s_{2}-1\right)} \mid m_{2} \geq 1\right\} \\
& \cup\left\{\widetilde{h}_{s_{1}} \widetilde{g}_{s_{2}}-\tilde{\chi}_{\psi\left(s_{1}+s_{2}\right)}\left(\tilde{f}_{\lambda}\right)^{\phi\left(s_{1}+s_{2}\right)} \mid m_{2}=0\right\} \\
& \cup\left\{\widetilde{h}_{s_{1}} \widetilde{h}_{s_{2}}+\left(\frac{n-1}{2}\right) \widetilde{g}_{\psi\left(s_{1}+s_{2}\right)}\left(\tilde{f}_{\lambda}\right)^{\phi\left(s_{1}+s_{2}\right)}\right\} \\
& \cup\left\{\tilde{\chi}_{s_{1}} \tilde{f}_{s_{2}}+\widetilde{\varepsilon}_{1} \widetilde{g}_{\psi\left(s_{1}+s_{2}-1\right)}\left(\tilde{f}_{\lambda}\right)^{\phi\left(s_{1}+s_{2}-1\right)} \mid m_{2} \geq 1\right\} \\
& \cup\left\{\widetilde{\varepsilon}_{1} \tilde{\chi}_{s}\right\} \cup\left\{\tilde{\chi}_{s_{1}} \widetilde{g}_{s_{2}}\right\} \cup\left\{\tilde{\chi}_{s_{1}} \tilde{h}_{s_{2}}\right\} \cup\left\{\tilde{\chi}_{s_{1}} \tilde{\chi}_{s_{2}}\right\} \text {. }
\end{aligned}
$$

Now, we pick the following set $\mathcal{E}_{i}$ of homogeneous elements of the algebra $k\left[\mathcal{X}_{i}\right]$, where $1 \leq i \leq 13$ :

$$
\begin{aligned}
\mathcal{E}_{1}= & \mathcal{E}_{\mathcal{A}} \cup\left\{\widetilde{p}_{s_{1}} \tilde{f}_{s_{2}}+\widetilde{g}_{\psi\left(s_{1}+s_{2}\right)}\left(\tilde{f}_{\lambda}\right)^{\phi\left(s_{1}+s_{2}\right)} \mid m_{2} \geq 1\right\} \\
& \cup\left\{\widetilde{p}_{s_{1}} \widetilde{p}_{s_{2}}+\left(\frac{n}{2}\right) \widetilde{g}_{\psi\left(s_{1}+s_{2}\right)}\left(\tilde{f}_{\lambda}\right)^{\phi\left(s_{1}+s_{2}\right)}\right\} ; \\
\mathcal{E}_{2}= & \mathcal{E}_{1} \cup \mathcal{E}_{\mathcal{D}} \cup\left\{\widetilde{\varepsilon}_{0}^{(j)} \widetilde{\varepsilon}_{1}, \widetilde{\varepsilon}_{0}^{(j)} \widetilde{f}_{s}, \widetilde{\varepsilon}_{0}^{(j)} \widetilde{g}_{s}, \widetilde{\varepsilon}_{0}^{(j)} \widetilde{p}_{s} \mid 1 \leq j \leq n\right\} ;
\end{aligned}
$$




$$
\begin{aligned}
& \mathcal{E}_{3}=\mathcal{E}_{1} \cup \mathcal{E}_{\mathcal{D}} \cup\left\{\widetilde{\varepsilon}_{0}^{(j)} \widetilde{f}_{s} \mid 1 \leq j \leq n-2, s<\lambda\right\} \\
& \cup\left\{\widetilde{\varepsilon}_{0}^{(q)} \widetilde{f}_{s}-\widetilde{\varepsilon}_{1} \widetilde{g}_{s-1} \mid q \in\{n-1, n\}, s<\lambda\right\} \\
& \cup\left\{\widetilde{\varepsilon}_{0}^{(j)} \widetilde{f}_{\lambda}-\widetilde{\varepsilon}_{1} \tilde{f}_{\lambda-1} \mid 1 \leq j \leq n-2\right\} \\
& \cup\left\{\widetilde{\varepsilon}_{0}^{(n-1)} \widetilde{f}_{\lambda}-\left(\frac{n-2}{2}\right) \widetilde{\varepsilon}_{1} \tilde{f}_{\lambda-1}-\widetilde{\varepsilon}_{1} \widetilde{p}_{\lambda-1}\right\} \\
& \cup\left\{\widetilde{\varepsilon}_{0}^{(n)} \tilde{f}_{\lambda}-\left(\frac{n}{2}\right) \widetilde{\varepsilon}_{1} \tilde{f}_{\lambda-1}-\widetilde{\varepsilon}_{1} \tilde{p}_{\lambda-1}\right\} \\
& \cup\left\{\widetilde{\varepsilon}_{0}^{(j)} \widetilde{p}_{s}-\widetilde{\varepsilon}_{1} \widetilde{g}_{s-1} \mid 1 \leq j \leq n-1\right\} \cup\left\{\widetilde{\varepsilon}_{0}^{(n)} \widetilde{p}_{s}\right\} \cup\left\{\widetilde{\varepsilon}_{0}^{(j)} \widetilde{g}_{s}, \widetilde{\varepsilon}_{0}^{(j)} \widetilde{\varepsilon}_{1} \mid 1 \leq j \leq n\right\} ; \\
& \mathcal{E}_{4}=\mathcal{E}_{1} \cup\left\{\tilde{\chi}_{s_{1}} \tilde{f}_{s_{2}}+\widetilde{\varepsilon}_{1} \widetilde{g}_{\psi\left(s_{1}+s_{2}-1\right)}\left(\tilde{f}_{\lambda}\right)^{\phi\left(s_{1}+s_{2}-1\right)} \mid m_{2} \geq 1\right\} \\
& \cup\left\{\widetilde{\varepsilon}_{1} \tilde{f}_{s}-2 \widetilde{\varepsilon}_{1} \tilde{p}_{s}\right\} \cup\left\{\widetilde{\varepsilon}_{1} \tilde{\chi}_{s}\right\} \cup\left\{\tilde{\chi}_{s_{1}} \widetilde{g}_{s_{2}}\right\} \cup\left\{\tilde{\chi}_{s_{1}} \tilde{p}_{s_{2}}\right\} \cup\left\{\tilde{\chi}_{s_{1}} \tilde{\chi}_{s_{2}}\right\} ; \\
& \mathcal{E}_{5}=\mathcal{E}_{4} \cup \mathcal{E}_{\mathcal{D}} \cup\left\{\widetilde{\varepsilon}_{0}^{(j)} \widetilde{\varepsilon}_{1}, \widetilde{\varepsilon}_{0}^{(j)} \widetilde{f}_{s}, \widetilde{\varepsilon}_{0}^{(j)} \widetilde{g}_{s}, \widetilde{\varepsilon}_{0}^{(j)} \widetilde{p}_{s}, \widetilde{\varepsilon}_{0}^{(j)} \widetilde{\chi}_{s} \mid 1 \leq j \leq n\right\} ; \\
& \mathcal{E}_{6}=\mathcal{E}_{\mathcal{A}} \cup\left\{\widetilde{h}_{s_{1}} \tilde{f}_{s_{2}} \mid m_{2} \geq 1\right\} \cup\left\{\widetilde{h}_{s_{1}} \widetilde{g}_{s_{2}}\right\} \\
& \cup\left\{\widetilde{h}_{s_{1}} \widetilde{h}_{s_{2}}+\left(\frac{n-1}{2}\right) \widetilde{g}_{\psi\left(s_{1}+s_{2}\right)}\left(\tilde{f}_{\lambda}\right)^{\phi\left(s_{1}+s_{2}\right)}\right\} ; \\
& \mathcal{E}_{7}=\mathcal{E}_{6} \cup \mathcal{E}_{\mathcal{D}} \cup\left\{\widetilde{\varepsilon}_{0}^{(j)} \widetilde{\varepsilon}_{1}, \widetilde{\varepsilon}_{0}^{(j)} \tilde{f}_{s}, \widetilde{\varepsilon}_{0}^{(j)} \widetilde{g}_{s}, \widetilde{\varepsilon}_{0}^{(j)} \widetilde{h}_{s} \mid 1 \leq j \leq n\right\} ; \\
& \mathcal{E}_{8}=\mathcal{E}_{6} \cup\left\{\tilde{\xi}_{s_{1}} \tilde{f}_{s_{2}} \mid m_{2} \geq 1\right\} \cup\left\{\widetilde{\varepsilon}_{1} \tilde{\xi}_{s}\right\} \cup\left\{\widetilde{\xi}_{s_{1}} \widetilde{g}_{s_{2}}\right\} \cup\left\{\tilde{\xi}_{s_{1}} \tilde{\xi}_{s_{2}}\right\} \\
& \cup\left\{\widetilde{\xi}_{s_{1}} \widetilde{h}_{s_{2}}+\widetilde{\varepsilon}_{1} \widetilde{g}_{\psi\left(s_{1}+s_{2}-1\right)}\left(\tilde{f}_{\lambda}\right)^{\phi\left(s_{1}+s_{2}-1\right)}\right\} \cup\left\{\widetilde{\varepsilon}_{1} \widetilde{h}_{s}\right\} ; \\
& \mathcal{E}_{9}=\mathcal{E}_{8} \cup \mathcal{E}_{\mathcal{D}} \cup\left\{\widetilde{\varepsilon}_{0}^{(j)} \widetilde{\varepsilon}_{1}, \widetilde{\varepsilon}_{0}^{(j)} \tilde{f}_{s}, \widetilde{\varepsilon}_{0}^{(j)} \widetilde{g}_{s}, \widetilde{\varepsilon}_{0}^{(j)} \widetilde{h}_{s}, \widetilde{\varepsilon}_{0}^{(j)} \widetilde{\xi}_{s} \mid 1 \leq j \leq n\right\} ; \\
& \mathcal{E}_{10}=\mathcal{E}_{\mathcal{A}} \cup \mathcal{E}_{\mathcal{A} \cup \mathcal{B}_{2} \cup \mathcal{C}_{1}} \\
& \cup\left\{\widetilde{g}_{s_{1}} \tilde{f}_{s_{2}}-\left(\frac{n-1}{2}\right)^{-1} \widetilde{\varepsilon}_{1} \tilde{h}_{\psi\left(s_{1}+s_{2}-1\right)}\left(\tilde{f}_{\lambda}\right)^{\phi\left(s_{1}+s_{2}-1\right)} \mid m_{1}+m_{2}=n-2\right\} ; \\
& \mathcal{E}_{11}=\mathcal{E}_{10} \cup \mathcal{E}_{\mathcal{D}} \cup\left\{\widetilde{\varepsilon}_{0}^{(j)} \tilde{f}_{s} \mid 1 \leq j \leq n-2\right\} \\
& \cup\left\{\widetilde{\varepsilon}_{0}^{(q)} \tilde{f}_{s}-\widetilde{\varepsilon}_{1} \widetilde{g}_{s-1} \mid q \in\{n-1, n\}, s<\lambda\right\} \\
& \cup\left\{\widetilde{\varepsilon}_{0}^{(q)} \tilde{f}_{\lambda}-\tilde{\chi}_{\lambda} \mid q \in\{n-1, n\}\right\} \cup\left\{\widetilde{\varepsilon}_{0}^{(j)} \widetilde{\varepsilon}_{1}, \widetilde{\varepsilon}_{0}^{(j)} \widetilde{g}_{s}, \widetilde{\varepsilon}_{0}^{(j)} \widetilde{h}_{s}, \widetilde{\varepsilon}_{0}^{(j)} \widetilde{\chi}_{s} \mid 1 \leq j \leq n\right\} ; \\
& \mathcal{E}_{12}=\mathcal{E}_{\mathcal{A}} \cup \mathcal{E}_{\mathcal{A} \cup \mathcal{B}_{2} \cup \mathcal{C}_{1}} \\
& \cup\left\{\widetilde{g}_{s_{1}} \tilde{f}_{s_{2}}-\widetilde{\xi}_{\psi\left(s_{1}+s_{2}\right)}\left(\tilde{f}_{\lambda}\right)^{\phi\left(s_{1}+s_{2}\right)} \mid m_{1}+m_{2}=n-2\right\} \\
& \cup\left\{\tilde{\xi}_{s_{1}} \tilde{f}_{s_{2}} \mid m_{2} \geq 1\right\} \cup\left\{\widetilde{\varepsilon}_{1} \tilde{\xi}_{s}\right\} \cup\left\{\tilde{\xi}_{s_{1}} \widetilde{g}_{s_{2}}\right\} \cup\left\{\tilde{\xi}_{s_{1}} \tilde{\xi}_{s_{2}}\right\} \cup\left\{\tilde{\xi}_{s_{1}} \tilde{\chi}_{s_{2}}\right\} \\
& \cup\left\{\widetilde{\xi}_{s_{1}} \widetilde{h}_{s_{2}}+\widetilde{\varepsilon}_{1} \widetilde{g}_{\psi\left(s_{1}+s_{2}-1\right)}\left(\widetilde{f}_{\lambda}\right)^{\phi\left(s_{1}+s_{2}-1\right)}\right\} \cup\left\{\widetilde{\varepsilon}_{1} \widetilde{h}_{s}\right\} ; \\
& \mathcal{E}_{13}=\mathcal{E}_{12} \cup \mathcal{E}_{\mathcal{D}} \cup\left\{\widetilde{\varepsilon}_{0}^{(j)} \tilde{f}_{s} \mid 1 \leq j \leq n-2\right\} \\
& \cup\left\{\widetilde{\varepsilon}_{0}^{(q)} \tilde{f}_{s}-\widetilde{\varepsilon}_{1} \widetilde{g}_{s-1} \mid q \in\{n-1, n\}, s<\lambda\right\} \\
& \cup\left\{\widetilde{\varepsilon}_{0}^{(q)} \tilde{f}_{\lambda}-\tilde{\chi}_{\lambda} \mid q \in\{n-1, n\}\right\} \\
& \cup\left\{\widetilde{\varepsilon}_{0}^{(j)} \widetilde{\varepsilon}_{1}, \widetilde{\varepsilon}_{0}^{(j)} \widetilde{g}_{s}, \widetilde{\varepsilon}_{0}^{(j)} \widetilde{h}_{s}, \widetilde{\varepsilon}_{0}^{(j)} \widetilde{\chi}_{s}, \widetilde{\varepsilon}_{0}^{(j)} \widetilde{\xi}_{s} \mid 1 \leq j \leq n\right\} .
\end{aligned}
$$

Finally, we denote by $I_{i}$ the ideal of the algebra $k\left[\mathcal{X}_{i}\right]$ generated by the set $\mathcal{E}_{i}$ for all $1 \leq i \leq 13$.

Theorem 4. 1) Suppose $2 \mid n$, char $k \nmid n-1$, and $r>1$. Then $\operatorname{HH}^{*}(R) \simeq k\left[\mathcal{X}_{1}\right] / I_{1}$.

2) Suppose $2 \mid n$, char $k \nmid n-1$, char $k \neq 2$, and $r=1$. Then $\operatorname{HH}^{*}(R) \simeq k\left[\mathcal{X}_{2}\right] / I_{2}$.

3) Suppose $2 \mid n$, char $k=2$, and $r=1$. Then $\operatorname{HH}^{*}(R) \simeq k\left[\mathcal{X}_{3}\right] / I_{3}$.

4) Suppose $2 \mid n$, char $k \mid n-1$, and $r>1$. Then $\mathrm{HH}^{*}(R) \simeq k\left[\mathcal{X}_{4}\right] / I_{4}$.

5) Suppose $2 \mid n$, char $k \mid n-1$, and $r=1$. Then $\mathrm{HH}^{*}(R) \simeq k\left[\mathcal{X}_{5}\right] / I_{5}$.

6) Suppose $2 \nmid n$, char $k \nmid\left(\frac{n-1}{2}\right)$, char $k \neq 2$, and $r>1$. Then $\mathrm{HH}^{*}(R) \simeq k\left[\mathcal{X}_{6}\right] / I_{6}$. 
7) Suppose $2 \nmid n$, char $k \nmid\left(\frac{n-1}{2}\right)$, char $k \neq 2$, and $r=1$. Then $\mathrm{HH}^{*}(R) \simeq k\left[\mathcal{X}_{7}\right] / I_{7}$.

8) Suppose $2 \nmid n$, char $k \mid\left(\frac{n-1}{2}\right)$, char $k \neq 2$, and $r>1$. Then $\mathrm{HH}^{*}(R) \simeq k\left[\mathcal{X}_{8}\right] / I_{8}$.

9) Suppose $2 \nmid n$, char $k \mid\left(\frac{n-1}{2}\right)$, char $k \neq 2$, and $r=1$. Then $\operatorname{HH}^{*}(R) \simeq k\left[\mathcal{X}_{9}\right] / I_{9}$.

10) Suppose $2 \nmid n$, char $k \nmid\left(\frac{n-1}{2}\right)$, char $k=2$, and $r>1$. Then $\mathrm{HH}^{*}(R) \simeq k\left[\mathcal{X}_{10}\right] / I_{10}$.

11) Suppose $2 \nmid n$, char $k \nmid\left(\frac{n-1}{2}\right)$, char $k=2$, and $r=1$. Then $\mathrm{HH}^{*}(R) \simeq k\left[\mathcal{X}_{11}\right] / I_{11}$.

12) Suppose $2 \nmid n$, char $k \mid\left(\frac{n-1}{2}\right)$, char $k=2$, and $r>1$. Then $\mathrm{HH}^{*}(R) \simeq k\left[\mathcal{X}_{12}\right] / I_{12}$.

13) Suppose $2 \nmid n$, char $k \mid\left(\frac{n-1}{2}\right)$, char $k=2$, and $r=1$. Then $\mathrm{HH}^{*}(R) \simeq k\left[\mathcal{X}_{13}\right] / I_{13}$.

Proof. In each case from 1) to 13), we consider the homomorphism of graded $k$-algebras $\pi: k\left[\mathcal{X}_{i}\right] \rightarrow \mathrm{HH}^{*}(R)$ that maps any element $\tilde{\theta}$ of the set $\mathcal{X}_{i}$ to the corresponding $\theta \in \mathrm{HH}^{*}(R)$. Corollary 2 shows that $\pi$ is a surjective homomorphism. From Propositions 35 and from (4.1) and (4.2), it follows that $I_{i} \subset \operatorname{Ker} \pi$ in each case among the 13 cases above. Consequently, there exists a surjective homomorphism of graded $k$-algebras $\bar{\pi}: k\left[\mathcal{X}_{i}\right] / I_{i} \rightarrow \mathrm{HH}^{*}(R)$ that maps the class of the element $\widetilde{\theta} \in \mathcal{X}_{i}$ to $\theta$. It remains to prove that $\bar{\pi}$ is an isomorphism. We denote the algebra $k\left[\mathcal{X}_{i}\right] / I_{i}$ by $A_{i}$. Let $A_{i}=\bigoplus_{s \geq 0} A_{i}^{s}$ be the decomposition of the algebra $A_{i}$ into homogeneous direct summands. Then it suffices to check that $\operatorname{dim}_{k} A_{i}^{s} \leq \operatorname{dim}_{k} \operatorname{HH}^{s}(R)$ for all $s \geq 0$. It is easily seen that any products of the form $\widetilde{\theta}_{1} \widetilde{\theta}_{2}$, where $\widetilde{\theta}_{1}$ and $\widetilde{\theta}_{2}$ are elements of $\mathcal{X}_{i}$ different from $\widetilde{\varepsilon}_{1}$ and $\widetilde{f}_{\lambda}$, are equal modulo the ideal $I_{i}$ either to 0 or to $\varkappa\left(\widetilde{\varepsilon}_{1}\right)^{a_{1}} \tilde{\theta}\left(\tilde{f}_{\lambda}\right)^{a_{2}}$, where $a_{1}, a_{2} \in\{0,1\}$, $\varkappa \in k$, and $\tilde{\theta} \in\left(\mathcal{X}_{i} \backslash\left\{\varepsilon_{1}, f_{\lambda}\right\}\right) \cup\{1\}$. Then we can reduce every nonzero product of the form $\widetilde{\theta}_{1} \ldots \widetilde{\theta}_{t}$, where $\widetilde{\theta}_{1}, \ldots, \widetilde{\theta}_{t} \in \mathcal{X}_{i}$, to $\varkappa\left(\widetilde{\varepsilon}_{1}\right)^{a_{1}} \widetilde{\theta}\left(\tilde{f}_{\lambda}\right)^{a_{2}}$ with $\varkappa \in k, a_{1} \in\{0,1\}$, $a_{2} \geq 0$, and $\tilde{\theta} \in\left(\mathcal{X}_{i} \backslash\left\{\varepsilon_{1}, f_{\lambda}\right\}\right) \cup\{1\}$. Since all elements of the set $\mathcal{X}_{i}$ are of degree at most $\lambda$ and all elements of degree $\lambda$ except $\tilde{f}_{\lambda}$ give 0 in the product with $\widetilde{\varepsilon}_{1}$, it follows that every homogeneous element $\rho$ of degree $s>\lambda$ can be represented in the form $\rho=\varkappa \rho_{0}\left(\tilde{f}_{\lambda}\right)^{a}$, where $\varkappa \in k, a \geq 1$, and $\rho_{0}$ is a homogeneous element of degree $s-a \lambda \leq \lambda$ in $A_{i}$. Consequently, if $s>\lambda$, then $\operatorname{dim}_{k} A_{i}^{s} \leq \operatorname{dim}_{k} A_{i}^{s-a \lambda}$, where $s-a \lambda \leq \lambda$. In this case, we have $\operatorname{dim}_{k} \mathrm{HH}^{s}(R)=\operatorname{dim}_{k} \mathrm{HH}^{s-a \lambda}(R)$. Hence, it suffices to prove that $\operatorname{dim}_{k} A_{i}^{s} \leq \operatorname{dim}_{k} \operatorname{HH}^{s}(R)$ for $0 \leq s \leq \lambda$. From the above it follows that $A_{i}^{s}$ is generated by the elements of the form $\left(\widetilde{\varepsilon}_{1}\right)^{a} \widetilde{\theta}$, where $a \in\{0,1\}$ and $\widetilde{\theta} \in \mathcal{X}_{i} \cup\{1\}$. The elements of this form are said to be normal.

Next we should consider the cases where one among the conditions of Theorems 2 or 3. depending on the value of $r$, is satisfied for $s$, and also the case where none of the conditions of the corresponding theorem is satisfied for $s$. We see that the number of normal elements in $A_{i}^{s}$ not lying in $\mathcal{E}_{i}$ is equal to $\operatorname{dim}_{k} \operatorname{HH}^{s}(R)$ for all $1 \leq i \leq 13$ and $0 \leq s \leq \lambda$, except for the cases where $i$ equals 4 or 5 and $s=(l+1)(2 n-3)$ satisfies either (2.6) or (2.12). Moreover, in the remaining cases, the number of such elements is equal to $\operatorname{dim}_{k} \mathrm{HH}^{s}(R)+1$. Since $\widetilde{\varepsilon}_{1} \widetilde{f}_{s-1}-2 \widetilde{\varepsilon}_{1} \widetilde{p}_{s-1} \in \mathcal{E}_{i}$ in these cases, the number of linearly independent normal elements in $A_{i}^{s}$ does not exceed $\operatorname{dim}_{k} \mathrm{HH}^{s}(R)$. Hence, $\operatorname{dim}_{k} A_{i}^{s} \leq \operatorname{dim}_{k} \operatorname{HH}^{s}(R)$ for all $s \geq 0$. Thus, the theorem is proved.

\section{References}

[1] C. Riedtmann, Algebren, Darstellungsköcher, Überlagerungen und zurück, Comment. Math. Helv. 55 (1980), 199-224. MR0576602 (82k:16039)

[2] A. S. Dugas, Periodic resolutions and self-injective algebras of finite type, Preprint, 2008.

[3] C. Riedtmann, Representation-finite self-injective algebras of class $A_{n}$, Representation Theory, II (Proc. Second Internat. Conf., Carleton Univ., Ottawa, Ont., 1979), Lecture Notes in Math., vol. 832, Springer, Berlin, 1980, pp. 449-520. MR0607169 (82k:16040)

[4] K. Erdmann and T. Holm, Twisted bimodules and Hochschild cohomology for self-injective algebras of class $A_{n}$, Forum Math. 11 (1999), 177-201. MR1680594(2001c:16018) 
[5] K. Erdmann, T. Holm, and N. Snashall, Twisted bimodules and Hochschild cohomology for selfinjective algebras of class $A_{n}$. II, Algebr. Represent. Theory 5 (2002), 457-482. MR1935856 (2004a:16013)

[6] A. I. Generalov and M. A. Kachalova, Bimodule resolution of the Möbius algebra, Zap. Nauchn. Sem. S.-Peterburg. Otdel. Mat. Inst. Steklov. (POMI) 321 (2005), 36-66; English transl., J. Math. Sci. (N.Y.) 136 (2006), no. 3, 3850-3866. MR2138411 (2006a:16014)

[7] M. A. Kachalova, The Hochschild cohomology for the Möbius algebra, Zap. Nauchn. Sem. S.-Peterburg. Otdel. Mat. Inst. Steklov. (POMI) 330 (2006), 173-200; English transl., J. Math. Sci. (N.Y.) 140 (2007), no. 5, 699-715. MR2253573 (2007f:16016)

[8] Yu. V. Volkov, Stable equivalence classes of self-injective algebras of tree class $D_{n}$, Vestnik S.Peterburg. Univ. Ser. 1 2008, vyp. 1, 15-21; English transl., Vestnik St. Petersburg Univ. Math. 41 (2008), no. 1, 9-14. MR2406893 (2009c:16040)

[9] Yu. V. Volkov and A. I. Generalov, Hochschild cohomology for self-injective algebras of tree type $D_{n}$. I, Zap. Nauchn. Sem. S.-Peterburg. Otdel. Mat. Inst. Steklov. (POMI) 343 (2007), 121-182; English transl., J. Math. Sci. (N.Y.) 147 (2007), no. 5, 7042-7073. MR2469415(2010h:16024)

Department of Mathematics and Mechanics, St. Petersburg State University, Universitetskaya Ul. 28, Stary Petergof, St. Petersburg 198504, Russia

E-mail address: wolf86_666@list.ru

Received 15/MAY/2010

Translated by THE AUTHOR 